\title{
Singular vector decomposition of the internal variability of the Canadian Regional Climate Model
}

\author{
Emilia Paula Diaconescu • René Laprise • \\ Ayrton Zadra
}

Received: 30 September 2010/Accepted: 24 August 2011/Published online: 23 September 2011

(C) The Author(s) 2011. This article is published with open access at Springerlink.com

\begin{abstract}
Previous studies have shown that Regional Climate Models (RCM) internal variability (IV) fluctuates in time depending on synoptic events. This study focuses on the physical understanding of episodes with rapid growth of IV. An ensemble of 21 simulations, differing only in their initial conditions, was run over North America using version 5 of the Canadian RCM (CRCM). The IV is quantified in terms of energy of CRCM perturbations with respect to a reference simulation. The working hypothesis is that IV is arising through rapidly growing perturbations developed in dynamically unstable regions. If indeed IV is triggered by the growth of unstable perturbations, a large proportion of the CRCM perturbations must project onto the most unstable singular vectors (SVs). A set of ten SVs was computed to identify the orthogonal set of perturbations that provide the maximum growth with respect to the dry totalenergy norm during the course of the CRCM ensemble of simulations. CRCM perturbations were then projected onto the subspace of SVs. The analysis of one episode of rapid growth of IV is presented in detail. It is shown that a large part of the IV growth is explained by initially smallamplitude unstable perturbations represented by the ten
\end{abstract}

E. P. Diaconescu $(\bowtie) \cdot$ R. Laprise $\cdot$ A. Zadra

Department of Earth and Atmospheric Sciences, Canadian Network for Regional Climate Modelling and Diagnostics, University of Quebec at Montreal (UQAM), P.O. Box 8888, Succ. Centre-ville, Montreal, QC H3C 3P8, Canada e-mail: diacones@sca.uqam.ca

E. P. Diaconescu $\cdot$ R. Laprise $\cdot$ A. Zadra

Centre ESCER (Étude et Simulation du Climat à l'Échelle Régionale), Montreal, QC, Canada

A. Zadra

Meteorological Research Division, Environment Canada,

Montreal, QC, Canada leading SVs, the SV subspace accounting for over $70 \%$ of the CRCM IV growth in $36 \mathrm{~h}$. The projection on the leading $\mathrm{SV}$ at final time is greater than the projection on the remaining SVs and there is a high similarity between the CRCM perturbations and the leading SV after 24-36 h tangent-linear model integration. The vertical structure of perturbations revealed that the baroclinic conversion is the dominant process in IV growth for this particular episode.

Keywords Internal variability - Regional climate model · Singular vectors $\cdot$ Growing perturbations

\section{Introduction}

It is common knowledge that the climate system is a complex dynamical system with natural variability occurring on a wide range of temporal and spatial scales. The complex interactions between and within the different components of the climate system result in atmospheric disturbances with several temporal and spatial scales. While low-frequency fluctuations are induced in the atmosphere by similar fluctuations in the sea surface temperature, soil moisture or interactions with solar radiation, the high-frequency weather fluctuations are associated with nonlinear interactions within the atmosphere. Nonlinearity is a key ingredient of chaotic systems. An important characteristic of chaotic systems is their sensitivity to small differences in initial conditions. This feature has dramatic consequences on the system's predictability, as it will be further explained.

In order to determine the future state of the atmosphere, we use atmospheric climate models, which are mathematical and numerical simplified versions of the atmosphere 
that either operate on the entire globe (Atmospheric General Circulation Model-AGCM) or on limited regions of the world (Regional Climate Model-RCM). There are two inevitable problems with climate models. First, they are only approximations of the real climate system and therefore are never perfect. Second, these models require initial conditions (ICs) in order to start the simulations. The variables used to simulate the principal atmospheric processes are the temperature, wind, pressure and humidity, and it is impossible to know their exact values at each point in the atmosphere. Therefore the model's ICs always have errors. These two problems associated with the atmosphere's chaotic nature make it impossible to determine exactly the future state of the system beyond a few days, because initially small errors will grow causing the highfrequency part of the model solution to eventually differ from the real atmospheric variations (Lorenz 1963, 1965). Hence, running a model several times with only small differences in the ICs (reflecting the degree of uncertainty in analysis which is based on observations) will result in different solutions for the same prediction problem. The predictability of synoptic events is lost after a period of several days. In other words, the memory of ICs is lost: any one member become uncorrelated with another member in the same ensemble.

The dispersion of model's solutions caused by its sensitivity to small differences in ICs is named internal variability (IV). IV can be studied by constructing ensembles of simulations where everything (including boundary forcing) is the same, except ICs.

In numerical weather prediction (NWP), the interest is then to provide as accurate as possible information for the ICs. In order to address this issue, data assimilation techniques are used and large ensembles are specifically designed to sample the subspace of the potential errors in observations. Ensemble prediction systems (EPS) have been implemented operationally in the early 1990s at the National Centers for Environmental Prediction (NCEP; Toth and Kalnay 1997) and at the European Centre for Medium-Range Weather Forecasts (ECMWF; Molteni et al. 1996), and later in other centres such as at the Meteorological Service of Canada (MSC; Pellerin et al. 2003); see Descamps and Talagrand (2007) for an overview. Nowadays, there are four popular methods used for initialization of EPS: the methods of singular vectors (SV), bred modes, ensemble Kalman filter and ensemble transform Kalman filter (these last two methods being ensemble-based data assimilation approaches).

As stated before, the time evolution of any perturbations, no matter how weak they are, will eventually erase the memory of the initial values. Hence, at climate scales, the model's response to initial atmospheric perturbations is independent of the magnitude as well as the type of the perturbation. This behaviour has been verified and confirmed by Lucas-Picher et al. (2008a) who initialized the different members of a RCM ensemble by either varying the initialization time between the different members or by adding random or fixed perturbations in some of the atmospheric fields. For AGCM and RCM ensembles, it does not matter how the atmospheric ICs are perturbed. Usually the ensemble is constructed by offsetting the initialization time of each simulation by 6 or $24 \mathrm{~h}$. After a spin-up period, the IV in AGCM will reach the level of natural climate variability. The behaviour of nested RCM contrasts with that of global models, because the model lateral boundaries (MLB) exert some control on the perturbations' growth and will thus limit the IV. Vukicevic and Paegle (1989) showed that the one-way interacting boundary conditions enhance the predictability of the flow in a local region compared to the case without lateral boundary constraint. They pointed out that the degree of boundary constraint is a function of the size of the domain and the nature of the flow in the domain.

The issue of the IV in RCM has been investigated previously in several studies (e.g., Giorgi and Bi 2000; Rinke and Dethloff 2000; Christensen et al. 2001; Caya and Biner 2004; Rinke et al. 2004; Vanvyve et al. 2007; Alexandru et al. 2007; Lucas-Picher et al. 2008a; Šeparović et al. 2008; Rapaić et al. 2010). These studies point to the fact that the RCMs' IV depends on the domain's size and location, the atmospheric variable under study, and on the season, being conditioned by the synoptic situation. LucasPicher et al. (2008a) have studied the IV for a large domain covering the North American continent. They found that the IV of mean-sea-level pressure and screen temperature exhibits a weak annual cycle with a maximum occurring during spring, while the precipitation IV shows a stronger annual cycle peaking during the summer. In their study over a circumpolar Arctic domain, Rinke et al. (2004) found a different annual cycle for temperature with maxima in autumn and winter.

Another important feature of RCM IV is its dependence on the domain size. This characteristic was clearly documented in the study of Alexandru et al. (2007), who have compared the IV of an RCM for five different domain sizes on the East Coast of North America during summer of 1993. They found that the IV is weaker for a small domain than for a larger domain, the RCM solution being more strongly constrained by the MLB. Rinke and Dethloff (2000) and Rapaic et al. (2010) arrived at the same conclusion when studying the IV for different size domains over the Arctic and North Atlantic regions, respectively.

Rapaic et al. (2010) computed the IV separately for the large- and small-scale components of the spectrum, using a length scale of $1,400 \mathrm{~km}$ for separation. They showed that, for the temperature field, the IV is weaker for the large 
scales than for the small scales, and noted that the IV for precipitation, a variable that is not strongly constrained by the MLB, is much stronger than for temperature.

An additional factor that can influence the IV is the time an air parcel spends inside the RCM domain, with longer residence times favouring perturbation growth. In their study, Lucas-Picher et al. (2008b) showed that the IV for fields that are driven at the MLB (such as mean-sea-level pressure and screen temperature fields) was well correlated with the residence time. On the other hand, the correlation was very weak for precipitation, which is a more local process and is not driven at the MLB. Nevertheless, the strength of the mean flow influences the divergence of the solutions, through the time required for the perturbations to approach the MLB and exit the domain. As a consequence, the geographical location of the RCM domain also influences the IV. A configuration with a strong through flow will quickly drive the perturbations out of the domain and therefore the growth will be limited; on the other hand, episodes with recirculation flow will lead to high IV (e.g., Laprise et al. 2008). Rinke et al. (2004) found that the IV is substantially larger for a circumpolar Arctic domain than for middle-latitude domains of comparable sizes; they hypothesised that this is because the Arctic domain is characterized, especially in winter and autumn, by a weaker circulation through the MLB and by an axisymmetric vortex that impedes the perturbation from exiting the domain.

Nikiema and Laprise (2010) have established prognostic budget equations of the IV for the potential temperature and the relative vorticity fields in simulations with the Canadian RCM over a domain centred on the East Coast of North America, for summer 1993. The two equations present similar terms, notably terms relating to the transport of IV by ensemble-mean flow and to the covariance of fluctuations acting on the gradient of the ensemble-mean state. It was noted that episodes of large IV growth usually occur where and when the ensemblemean state is cyclonic. The analysis of the different components of the IV tendency for an episode with important IV in July 1993 revealed that, for that particular summer episode, the most important contribution to the potential temperature IV growth came from covariance of potential temperature and diabatic heating from convection and condensation. The fluctuation available potential energy, generated by condensation and convection processes, was next converted into fluctuation kinetic energy. For the relative vorticity IV tendency, the most important terms were associated with horizontal components, emphasizing the importance of the horizontal flow in the vorticity IV tendency of this particular episode of high IV. Their study was focused on a summer case of high IV associated with a high synoptic activity. We hypothesize that the results may be different if the analysis is focused on a winter case of IV growth.

All these studies point to the fact that the IV does not have a long-term trend, but is rather characterized by episodes of rapid growth and decay depending on the synoptic situation. The divergence of the RCM solutions appears to be the result of the competition between two processes. On one side there are perturbations that are excited by the instabilities of the climate system. On the other side there is the forcing exerted by the lateral boundaries of the nested model that encourages the simulations of the RCM toward the same solution; the force of this forcing is given by the strength of the mean flow through the domain, which is strongly dependent on location, season, and domain size. Finally, everything is modulated by the synoptic conditions: periods with important synoptic activity will favour divergence growth, while calm synoptic periods will be characterized by a convergence of the solutions.

In spite of the many studies dedicated to IV, a very small number of them are focused on the physical reasons of its development. It is of great interest to elucidate the dynamical processes responsible for the episodic growth and decay of IV, especially in wintertime when the MLB exercise an important forcing. In this paper we focus on the physical understanding of episodes of rapid growth of IV in simulations of the Canadian Regional Climate Model (CRCM) for December 1992. Our working hypothesis is that IV growth results from rapidly growing perturbations through hydrodynamical instabilities within the regional domain. As a consequence, in periods with large instability the atmosphere should behave more chaotically and small differences would amplify rapidly, while in calm synoptic periods the ensemble members should stay within a narrow range and the IV would have smaller values.

A possible way to approach the issue of hydrodynamical instability is through the singular vector (SV) technique. The SVs from a linearized model provide the most rapidly growing perturbations over a chosen time interval with respect to a given norm. SVs have been used in various applications such as observation targeting (e.g., Buizza and Montani 1999; Barkmeijer et al. 2001; Wu et al. 2009) and especially in ensemble prediction for generating ICs for NWP EPS. By sampling the phase space in the most unstable directions, the ensemble is supposed to account for a maximum fraction of pdf variance and give to sufficient spread for the ensemble (e.g., Molteni et al. 1996). Further background on SVs is given in Sect. 2.

Numerous studies have emphasized the capability of SVs to capture atmospheric regions and periods characterized by large hydrodynamical instability (e.g., Borges and Hartmann 1992; Palmer et al. 1994). Our study aims at evaluating whether episodes of important IV growth can be characterised in terms of SVs. In order to address this issue, 
a CRCM ensemble of simulations is considered, made of a reference simulation and a set of perturbed member simulations from the reference. We aim at providing a systematic comparison between the CRCM perturbations and the SVs computed using the atmospheric conditions taken from the reference simulation during an episode of important IV growth. Given the linear nature of SVs, this comparison will also provide information concerning the relative roles played by linear and nonlinear processes in the IV growth.

In the following we will first present some general aspects regarding the SV technique (Sect. 2). We will then present the experiment setup with short descriptions of the models in Sect. 3. The main results concerning the CRCM IV, the computation of CRCM perturbations and their comparison with one set of SVs will be presented in Sect. 4. Finally, Sect. 5 will summarize the main findings.

\section{Singular vector technique}

The SV theory rests on the assumption that the evolution of small perturbations over a short time interval can be described to a good degree of approximation by a linearized system, named the tangent-linear model (TLM).

Consider $\mathbf{X}(\mathrm{t})$ the state vector, which includes fields such as temperature, wind and surface pressure, and assume that its evolution in time can be described by the equation

$\frac{d \mathbf{X}}{d t}=\mathbf{F}(\mathbf{X})$.

where $\mathbf{F}(\mathbf{X})$ represents the nonlinear model tendency. According to the linear theory, the evolution of a small perturbation $x(t)$ from the state vector may be approximately described by

$\frac{d x}{d t}=\mathbf{A}_{F} x$,

where $\left.\mathbf{A}_{F} \equiv \frac{d \mathbf{F}}{d \mathbf{X}}\right|_{\mathbf{X}(t)}$ represents the TLM relative to the trajectory $\mathbf{X}(\mathrm{t})$. Hence, we can use the TLM to describe the time evolution of infinitesimal-amplitude perturbations superimposed on a fully developed, nonlinear state of a system. The integration of TLM, named the propagator of the TLM, $\mathbf{L}\left(t, t_{0}\right)$, maps initially small perturbations $x\left(t_{0}\right)$ from time $t_{0}$ (henceforth referred to as the initial time) to a perturbation at later time $t$ (referred to as the final time) $(x(t))$ :

$x(t)=\mathbf{L}\left(t, t_{0}\right) x\left(t_{0}\right)$.

Because it is linearized around the detailed flow from $t_{0}$ to $t$, the TLM depends on the solution of the nonlinear model (the basic state), but it does not depend on the perturbation (Kalnay 2002). Perturbation growth can be quantified by calculating its amplitude at initial and final times measured by a chosen norm:

$$
\begin{aligned}
& \left\|x\left(t_{0}\right)\right\|_{E_{0}}^{2}=\left\langle x\left(t_{0}\right) ; E_{0} x\left(t_{0}\right)\right\rangle \\
& \|x(t)\|_{E_{t}}^{2}=\left\langle x(t) ; E_{t} x(t)\right\rangle
\end{aligned}
$$

Here, $\langle$,$\rangle denotes the Euclidean inner product, E_{0}$ represents the initial-time norm and $E_{t}$ the final-time norm.

$\mathrm{SVs}$ are defined as the perturbations that, under linearized dynamics about a time-evolving basic flow, grow most rapidly over a given time interval known as "optimization time interval", according to a given norm. SVs can be obtained by solving the generalized eigenvalue problem

$\mathbf{L}^{*} E_{t} \mathbf{L} y_{i}\left(t_{0}\right)=\lambda_{i}^{2} E_{0} y_{i}\left(t_{0}\right)$

where $\mathbf{L}^{*}$ is the adjoint of the operator $\mathbf{L}, \lambda$ is the singular value, and $y_{i}\left(t_{0}\right)$ is the initial-time $\mathrm{SV}$. Hence, the problem of finding the perturbations with maximum growth in terms of a norm $E$, that is the singular vectors, can be reduced to the search of the eigenvectors of the matrix $\mathbf{E}_{0}^{-1 / 2} \mathbf{L}^{*} E_{t} \mathbf{L} \mathbf{E}_{0}^{-1 / 2}$ with the largest eigenvalues $\lambda^{2}$. Note that the left-hand side of the equation involves one integration with the TLM, followed by one integration with the adjoint model. The equation can be solved efficiently using software packages such as the Arnoldi Package (ARPACK).

The resulting SVs form an orthogonal set and can be ordered according to growth rate, with the fastest growing structure being the first singular vector. The first SV, also referred to as the leading $\mathrm{SV}$ and noted $S V 1$, is the SV that maximizes the amplification factor defined as the ratio between the final and the initial norms. This ratio is equal to the square of the singular value:

$\frac{\|x(t)\|^{2}}{\left\|x\left(t_{0}\right)\right\|^{2}}=\frac{\left\langle x(t), E_{t} x(t)\right\rangle}{\left\langle x\left(t_{0}\right), E_{0} x\left(t_{0}\right)\right\rangle}=\frac{x^{T}\left(t_{0}\right) \mathbf{L}^{*} E_{t} \mathbf{L} x\left(t_{0}\right)}{x^{T}\left(t_{0}\right) E_{0} x\left(t_{0}\right)}=\lambda^{2}$.

Once the initial-time SVs are obtained, the corresponding final-time SVs can be derived by integrating the TLM.

The SVs associated with the largest $\lambda$ are also called leading SVs. Given an orthogonal and complete set of SVs ordered according to amplification factor, any initial perturbation can be decomposed in terms of the complete set of SVs comprising growing, neutral and decaying solutions:

$x\left(t_{0}\right)=\sum_{j=1}^{N} \alpha_{j} \hat{y}_{j}\left(t_{0}\right)$

where $\hat{y}_{j}\left(t_{0}\right)$ are the initial-time SVs normalized to have the unity norm, and $\alpha_{j}=\left\langle x\left(t_{0}\right), E_{0} \hat{y}_{j}\left(t_{0}\right)\right\rangle$ are the respective projection coefficients. Because the singular vectors 
are orthonormal, the amplitude of the initial-time perturbation is

$\left|x\left(t_{0}\right)\right|=\sqrt{\left\|x\left(t_{0}\right)\right\|^{2}}=\sqrt{\sum_{j=1}^{N} \alpha_{j}^{2}}$,

and its amplitude at time $t$ is

$|x(t)|=\sqrt{\|x(t)\|^{2}}=\sqrt{\sum_{j=1}^{N} \alpha_{j}^{2} \lambda_{j}^{2}}$.

Therefore the final-time magnitude of the perturbation depends on the projection coefficients $\alpha_{j}^{2}$ weighted by the individual amplification factors $\lambda_{j}^{2}$. Hence, growth is expected only if the magnitudes of the projections on the growing SVs $\left(\lambda_{j}^{2}>1\right)$ are sufficiently large with respect to those for decaying SVs $\left(\lambda_{j}^{2}<1\right.$ ) (Ehrendorfer and Errico 1995; Errico et al. 2001).

The dimension of the model gives the number of SVs. However, in the most part of previous studies, truncated bases of SVs are used instead of the complete base. In the particular case of a growing perturbation, and for a truncated base with a small number of SVs, if only a few singular values are large, it is expected that the projection onto the leading SVs will dominate (Hartmann et al. 1995). On the other hand, if the number of SVs is very large, the overall projection onto the slow growing and decaying SVs could overwhelm the projection onto the leading SVs (Errico et al. 2001). For example, Errico et al. (2001) have used 13 sets with $100 \mathrm{SVs}$. They found that only small fractions (approximately 1\%) of the possible number of SVs are fast growing structures; the others are very slowly growing structures. In our analysis, we make the hypothesis that the CRCM ensemble perturbations, in periods of growing IV, are fast-growing perturbations that may be efficiently described by a subset of the 10 leading SVs, the remaining slowly-growing SVs being of little interest in our case.

\section{Experimental set-up}

The IV of an RCM is studied through an ensemble approach. An ensemble of simulations performed with a single version of a regional model with different initialization times and driven by the same set of lateral boundary conditions can be considered as a sample of the RCM solution space (e.g., Christensen et al. 2001).

\subsection{The Canadian Regional Climate Model ensemble}

In the present work, the IV is explored by constructing an ensemble of 21 integrations with version 5 of the Canadian
Regional Climate Model (CRCM_5), developed by the Canadian Regional Climate Modelling and Diagnostics Network in collaboration with the Meteorological Service of Canada (MSC) (Zadra et al. 2008). CRCM_5 is a limited-area version of the Global Environmental Multiscale (GEM) model employed for numerical weather prediction at the MSC (Côté et al. 1998; Yeh et al. 2002). The model solves the fully elastic nonhydrostatic equations with a hybrid vertical coordinate based on terrain-following normalized hydrostatic pressure (Laprise 1992), $\eta=$ $\left(p-p_{\text {top }}\right) /\left(p_{s}-p_{\text {top }}\right)$, where $p_{\text {top }}$ and $p_{s}$ are the pressure at the model top and at the surface, respectively. However, in the configuration we used, the model was run in hydrostatic mode. In our experiments, the model includes 53 irregularly spaced hybrid levels in the vertical between the surface and $10 \mathrm{hPa}$, and a limited-area, rotated, latitudelongitude mesh with $120 \times 120$ grid points with spacing of 0.5 degree, or about $55 \mathrm{~km}$. The time step is $30 \mathrm{~min}$. A ten grid-point wide Davies-type lateral boundary relaxation zone (Davies 1976) is applied. No large-scale spectral nudging (Alexandru et al. 2009) has been performed in this study. The integration area, shown in Fig. 1, is centred on the North American continent.

In our configuration, the model uses the Interactions Soil-Biosphere-Atmosphere (ISBA) as land-surface scheme (Bélair et al. 2003), the Kain-Fritsch scheme (Kain and Fritsch 1990) for deep convective processes, the Kuo transient scheme for shallow convection (Kuo 1965; Bélair et al. 2005) and the Sundqvist scheme (Sundqvist et al. 1989) for large-scale condensation. The radiation package

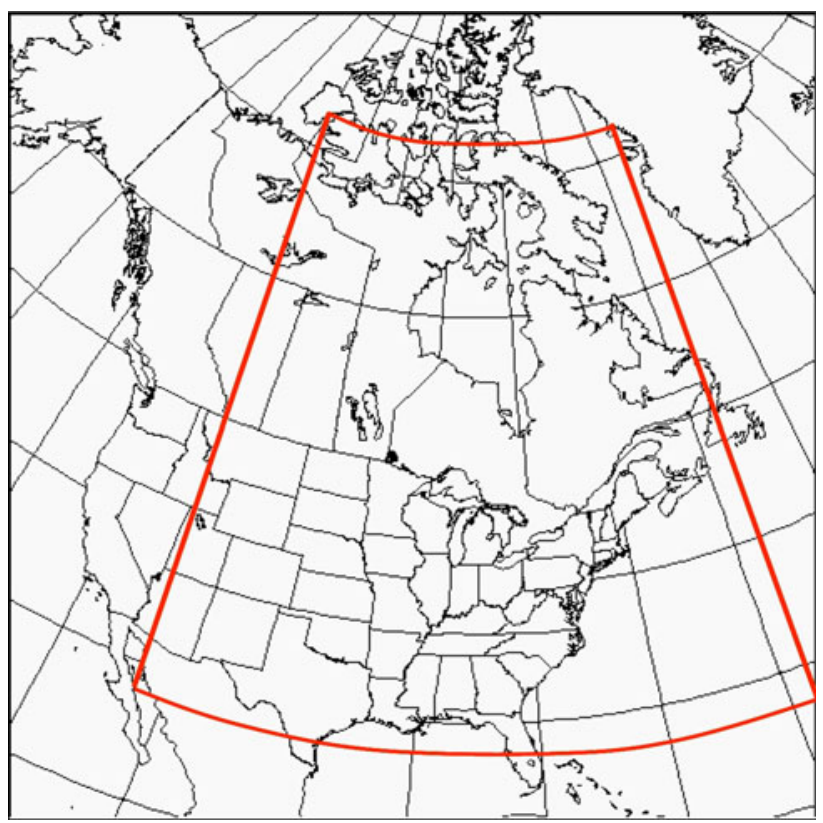

Fig. 1 CRCM integration domain. The red line shows the initial- and final-norm domain 
for solar and terrestrial radiation is based on the correlated$\mathrm{K}$ approach ( $\mathrm{Li}$ and Barker 2005). Subgrid-scale orographic gravity-wave drag is due to McFarlane (1987) and low-level orographic blocking is described in Zadra et al. (2003). Ocean surface conditions are prescribed. Sea surface temperatures (SST) and sea-ice are interpolated from the Atmosphere Model Intercomparison Project (AMIP2; Gleckler 1996) available on a one-degree latitude-longitude grid for monthly mean values.

Initial and lateral boundary conditions have been taken from the ECMWF (European Centre for Medium-range Weather Forecasts) reanalysis project ERA40 (Uppala et al. 2005) available every $6 \mathrm{~h}$.

Using the ensemble described here, we investigate the IV for December 1992. The 21 simulations have the same set-up except for the ICs that correspond to different starting dates between October 23 to November 12 at 00:00 UTC. The corresponding 21 runs are noted as $N A 23, N A 24, \ldots, N A 31, N A 01, \ldots, N A 11, N A 12$, the number representing the day of the ICs. Model output was archived every $6 \mathrm{~h}$ for each simulation. Because the simulations use the same driving fields and the same surface fields in the ICs, which are interactive afterwards, there are no lateral and lower boundary conditions perturbations. By design, the dispersion of model simulations is due only to the differences in the atmospheric initial conditions.

The first episode in the month of December 1992 when the IV begins to grow substantially in our ensemble of simulations occurred on December 4th (Fig. 2). Amongst the 21 members, the member named NAII was the closest one to the ERA40 driving data; for this reason we chose that member as the CRCM reference simulation for computing the IV.

\subsection{The SVs set}

The software required for the calculation of the SVs on a limited-area domain was not yet ready at the time of our investigation. Hence the SVs were computed using the tangent-linear (TLM) and adjoint versions of global GEM model, originally developed for 4D variational data assimilation system (Gauthier et al. 2007).

According to Eq. 5, several choices must be made when SVs are computed, such as the optimization time interval (OTI) and the initial and final norms. We computed several sets of SVs with different set-ups, but in the present paper we present only one set. Results for the other sets are qualitatively similar. The set presented in this paper has the following characteristics.

Since our analysis is focused on a winter period, we opted for TLM with a simplified physics containing only

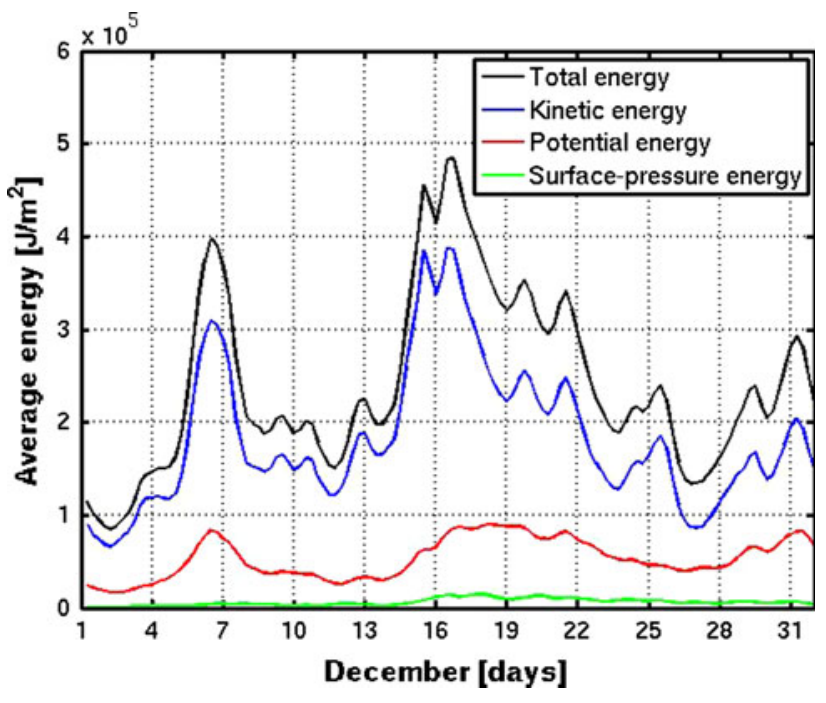

Fig. 2 Time evolution over the month of December 1992 for CRCM (black line) total average energy and its components: (blue line) kinetic, (red line) potential and (green line) surface-pressure energy

the vertical diffusion scheme. A detailed description of the available simplified parameterizations and their impact on SVs computed with the total-energy norm can be found in Zadra et al. (2004). The calculation of SVs is restricted to perturbations in horizontal wind $(\mathbf{V}=(u, v))$, temperature $(T)$ and surface pressure $\left(p_{s}\right)$ fields.

The evolution trajectory was generated using the nonlinear full-physics global GEM model starting from 4 December 1992 at 12:00 UTC, which represents the moment when the CRCM IV begins to grow. The corresponding ICs for the global model were constructed by combining the reference CRCM simulation (NA11) over the region encompassed by the CRCM integration, with those of the ERA40 reanalysis elsewhere on the globe.

The nonlinear, tangent-linear and adjoint global models were run with uniform horizontal resolution of $1^{\circ}, 28$ eta levels and a time step of $30 \mathrm{~min}$. The OTI is $36 \mathrm{~h}$, which corresponds to period of initial growth of the CRCM IV. Due to limits of computational cost, the number of SVs was restricted to the leading ten, and output every $6 \mathrm{~h}$.

As initial and final norms, we opted for the dry totalenergy norm restricted to a sub-domain within the CRCM integration area (the region delimited by the red line in Fig. 1), which permits the selection of SVs situated within the active region of CRCM simulations. The dry totalenergy norm is a rather common choice in the computation of singular vectors, and it provides a relative weighting between the mass $\left(T, p_{s}\right)$ and wind $(u, v)$ fields (Buehner and Zadra 2005). Here, it is defined by 


$$
\begin{aligned}
\langle x, E x\rangle= & \iint_{A}\left[\int\left(u^{2}+v^{2}\right) \frac{1}{g} \frac{\partial p}{\partial \eta} d \eta\right] d A \\
& +\iint_{A}\left[\int\left(\frac{c_{p}}{T_{r}} T^{2}\right) \frac{1}{g} \frac{\partial p}{\partial \eta} d \eta\right] d A \\
& +\iint_{A}\left[\frac{R T_{r}}{p_{r} g} p_{s}^{2}\right] d A
\end{aligned}
$$

where $A$ is the horizontal domain, $g$ is the gravity constant $\left(=9.806 \mathrm{~m} \mathrm{~s}^{-2}\right), \quad R$ is the gas constant for dry air $\left(=287.04 \mathrm{~J} \mathrm{~K}^{-1} \mathrm{~kg}^{-1}\right), c_{p}$ is the specific heat for dry air at constant pressure $\left(=1005.46 \mathrm{~J} \mathrm{~K}^{-1} \mathrm{~kg}^{-1}\right), T_{r}$ is a reference temperature $(=300 \mathrm{~K})$ and $p_{r}$ is a reference pressure $(=1,000 \mathrm{hPa})$. Here $x=\left(u, v, T, p_{s}\right)$ represents a perturbation of the model state vector comprising horizontal wind, temperature and surface pressure perturbations. The three terms on the right-hand side represent the kinetic, potential and surface-pressure components of the dry total energy, respectively. The vertical integral is restricted to the levels situated between hybrid levels $\eta=1$ and $\eta=0.1$, the levels above approximately $100 \mathrm{hPa}$ being neglected in order to eliminate potential growing modes, likely numerical in origin, that may be restricted to the top few model levels.

Hence, SVs are available in the form of perturbations in wind, temperature and surface pressure fields every $6 \mathrm{~h}$ on a grid-spacing of $1^{\circ}$ and 28 eta levels. In order to facilitate the comparison with the CRCM fields, the SVs are interpolated on the $0.5^{\circ} \mathrm{CRCM}$ grid and all the results are calculated with respect to the dry total-energy norm defined over the CRCM domain. The comparison between CRCM perturbations and $\mathrm{SVs}$ is based on their spatial structure, as well as their temporal evolution, partition and distribution of energy. We also compute the projection of CRCM perturbations on the set of SVs, which provides a quantitative measure of their similarity.

\section{Results}

Results are presented in three subsections. The first subsection describes the CRCM IV, the second is focused on the set of SVs and a comparison with the CRCM perturbations, and the last is dedicated to the projection of the CRCM perturbations on the set of SVs.

\subsection{CRCM internal variability}

The $I V$ is defined as the spread between the members in an ensemble with respect to a reference state:

$\sigma_{I V}^{2}(x, y, z, t)=\frac{1}{M-1} \sum_{m=1}^{M}\left[x_{m}^{\prime}(x, y, z, t)\right]^{2}$
Here, $M$ corresponds to the total number of members in the ensemble of simulations, $x_{m}^{\prime}(x, y, z, t)$ represents the perturbation of member $m\left(X_{m}\right)$ for fields $\left(u, v, T, p_{s}\right)$ function of time and location on the three-dimensional model grid,

$x_{m}^{\prime}=X_{m}-X_{r e f}$,

and $X_{\text {ref }}$ represents the reference state. In several other studies, $I V$ has been defined using the ensemble mean $(E M)$ as reference

$X_{\text {ref }}=\bar{X}^{M}=\frac{1}{M} \sum_{m=1}^{M} X_{m}$

(e.g., Alexandru et al. 2007; Šeparović et al. 2008). It is important however to realise that the $E M$ is in general not a solution of the atmospheric equations of motion. Also, the $E M$ lacks the fine scales that tend to characterise the irreproducible components, in the nomenclature of Šeparović et al. (2008). Because we will also want to use $X_{\text {ref }}$ as the trajectory in the calculation of the SVs, it must correspond to a solution of the equations of motion. In the following we have opted to use one member in the ensemble as reference; the member was chosen as the one closest to the driving conditions during the episode under study. For our case this corresponded to NA11, as mentioned earlier.

We mention that, in the case of an AGCM, the spread of an ensemble is very large approaching the value of natural transient variability. As a consequence, the ensemble members will be very different and can have different stability characteristics. However, in the case of a RCM, the spread of an ensemble is limited by the constraint exerted by the model lateral boundaries, which force all members towards the same solution as the driving field in the region of lateral boundaries. Therefore, the RCM members do not have time to diverge very much and will present similar features. The differences between the members of the RCM ensemble can hence be regarded in this case as small perturbations from a time-evolving reference state.

In this study we have chosen to express the IV in terms of energy. For each perturbation $x_{m}^{\prime}\left(u_{m}, v_{m}, T_{m}, p_{s m}\right)$ we have computed the perturbation total energy $\left(E_{m}\right)$ equivalent to the SV dry total-energy norm (Eq. 10). The ensemble-average energy of these perturbations provides an equivalent measure for the IV:

$$
\begin{aligned}
E_{m}= & \iiint_{V} \rho\left(u_{m}^{2}+v_{m}^{2}\right) d V o l+\iiint_{V} \rho\left(\frac{c_{p}}{T_{r}} T_{m}^{2}\right) d V o l \\
& +\iiint_{A}\left(\frac{R T_{r}}{p_{r} g} p_{s m}^{2}\right) d A ; E_{n}=\frac{1}{M-1} \sum_{\substack{m=1 \\
m \neq n}}^{M} E_{m}
\end{aligned}
$$

where $n$ indicates the reference member. 
Figure 2 shows the time evolution of the CRCM IV during the month of December 1992, expressed in terms of the total energy of perturbations. Note the episodic character of the IV, with various episodes of growth resulting in large IV. In this study we focused on the first maximum reached by 6 December at 12:00 UTC. Additional information is provided by the energy partition into kinetic, potential and surface-pressure terms, their time evolution is also shown in Fig. 2. During the entire period, most of the energy is in the kinetic form, with the surface-pressure term being relatively small. At about 12:00 UTC on 6 December, both the kinetic and potential energy components exhibit a maximum.

Figure 3 displays the horizontal distribution of the vertically integrated energy of the CRCM-perturbations, together with the 500-hPa geopotential field from the reference simulation. On 4 December at 12:00 UTC (Fig. 3a), some perturbations were present in two distinct regions of the domain: one over central Canada and another one over Québec-Labrador, close to the eastern boundary of the domain. As time progressed, the latter perturbation diminished and eventually disappeared, while the centraldomain perturbation kept growing and moved toward the East Coast of the USA (Fig. 3f). By 00:00 UTC on 6 December (Fig. 3e), the total energy distribution exhibits a large elongated maximum extending from south of the Great Lakes to the Atlantic Coast, close to the model's eastern lateral boundary. The presence of the boundary inhibits the further development eastward because the driving boundary fields in the atmospheric variables $(u, v, T)$ are the same for all simulations. As a consequence, a gradual decrease in the IV is noted close to the MLB, and the IV is actually zero in the boundaries relaxation zone. The peak in IV intensity is reached by 12:00 UTC 6 December and comes essentially from the maximum located south of the Great Lakes.

\subsection{CRCM perturbations versus SVs}

This section compares the CRCM IV perturbations with a set of ten leading SVs, computed as described in Sect. 2. The initial time for SVs corresponds to 4 December at 12:00 UTC when the CRCM IV begins to grow. In order to avoid the influence of the MLB on CRCM IV, the final time for the computation of the SVs was chosen to be 6 December at 00:00 UTC. The extent of final- and initialtime norms is within the CRCM domain, as shown in Fig. 1.

\subsubsection{Time evolution of energy}

The total-energy evolution of the ten leading SVs during the 36-h period, 12:00 UTC 4 December-00:00 UTC 6
December is shown in Fig. 4. For each SV, the energy was normalized by its initial-time value, i.e. it is set to $1 \mathrm{Jm}^{-2}$ at the initial time. Note that all ten leading SVs are growing perturbations, with the first leading SV having an energy amplification factor greater than 110 .

Some details on the perturbation growth can be found in the energy partition during the growth period; this is shown in Figs. 5 and 6. At the initial time, most of the SV total energy is in the form of potential energy while later, kinetic energy dominates; this is a typical feature of baroclinic disturbances that are usually captured by extratropical SVs. Figure 6a, b display the energy partition at initial and final time and show all ten SVs exhibit similar properties. The initial-time energy partition of SVs is different from that of CRCM perturbations. This suggests that the SVs are outside of the attractor at initial time (Kalnay 2002). However, in only one step, the dominance of energy components is reversed. Hence, with the exception of the initial time, the other steps are characterised by the dominance of kinetic energy - as in the case of CRCM perturbations (Fig. 2). This rapid change in the SV energy partition toward the CRCM-perturbation partition can be interpreted as a rapid (one time only) rotation of the initial-time SVs toward the system attractor (e.g., Szunyogh et al. 1997; Kalnay 2002). By December 6th 00:00 UTC, the ratio of kinetic energy to potential energy for the CRCM perturbations varies between 3.58 (for NA28) and 4.14 (for NA08 and NA10). For the set of SVs at final time, the ratio has values varying from 3.3 to 4.7. Also, the time evolution during the growing period shows that the CRCM-perturbation kinetic energy increases by a larger rate than the potential energy, as is the case for SVs.

\subsubsection{Structure of the leading SV}

The horizontal and vertical structures of the leading SV, normalized to have unit energy at initial time as explained earlier, is presented in terms of temperature perturbations in Fig. 7a-d, meridional wind in Fig. 8a-d, and zonal wind in Fig. 9a-d. The structure at initial time is presented in the panels (a) and (b), while the panels (c) and (d) show the evolved structures according to the TLM after $30 \mathrm{~h}$. At initial time, the leading SV has the structure of a wavepacket located in the centre of the domain, with perturbation maxima of $T=0.07^{\circ} \mathrm{C}, v=0.07 \mathrm{~m} / \mathrm{s}$ and $u=0.04 \mathrm{~m} / \mathrm{s}$. After $30 \mathrm{~h}$, the perturbation has moved southeast and reached the USA East Coast and Atlantic Ocean. The horizontal scale of the wavepacket has since grown considerably, and the amplitude maxima have grown to reach values of $T=0.2^{\circ} \mathrm{C}, v=0.48 \mathrm{~m} / \mathrm{s}$ and $u=0.35 \mathrm{~m} / \mathrm{s}$. Note once more that the amplification of the wind field is larger than that of the temperature field. 
(a) 1200 UTC 4 December 1992

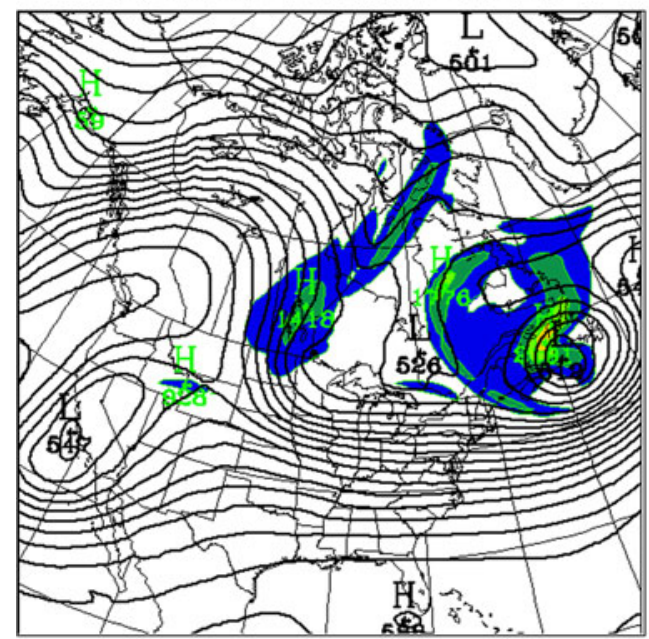

(c) 1200 UTC 5 December 1992

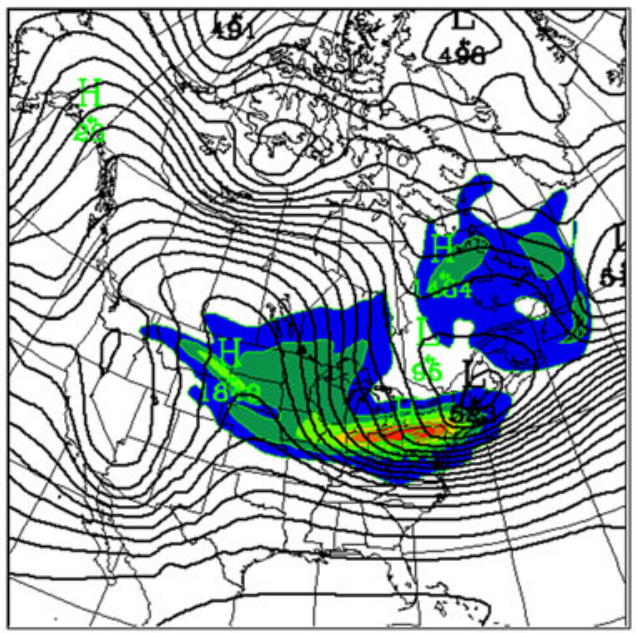

(e) 0000 UTC 6 December 1992

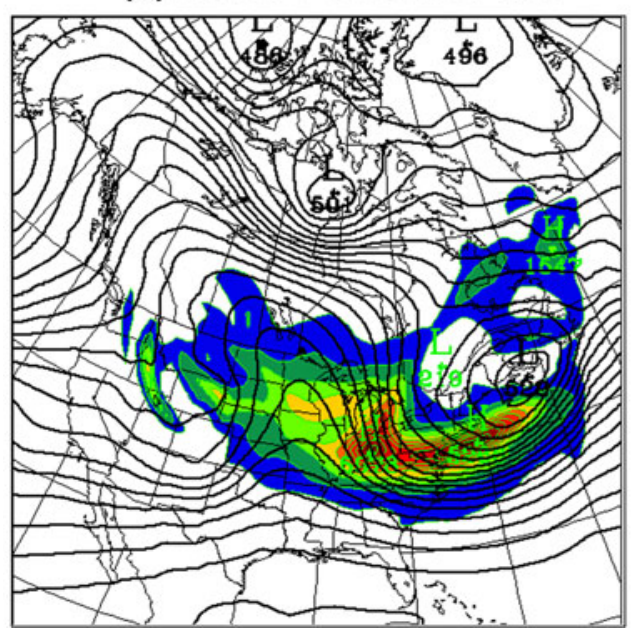

Fig. $3500 \mathrm{hPa}$ geopotential field (in dam) for the reference simulation and the average CRCM-perturbation total energy (in $\mathrm{kJ} / \mathrm{m}^{2}$ ) integrated between $100 \mathrm{hPa}$ and surface. The geopotential field (black (b) 0000 UTC 5 December 1992

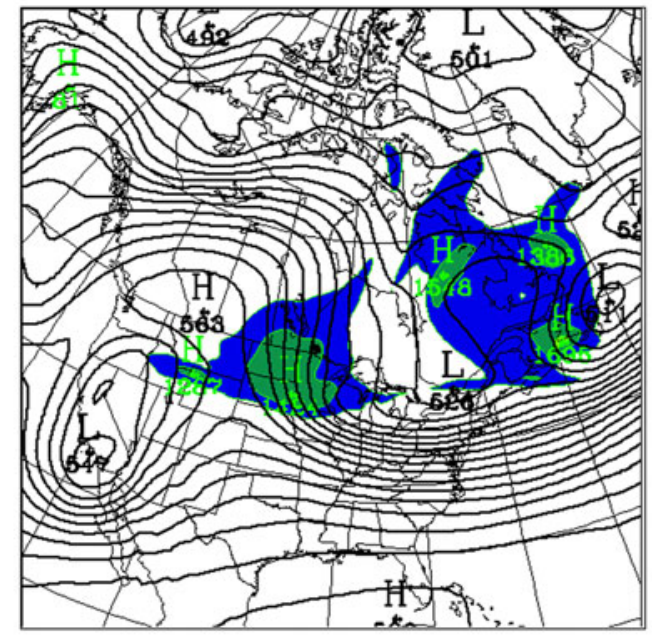

(d) 1800 UTC 5 December 1992

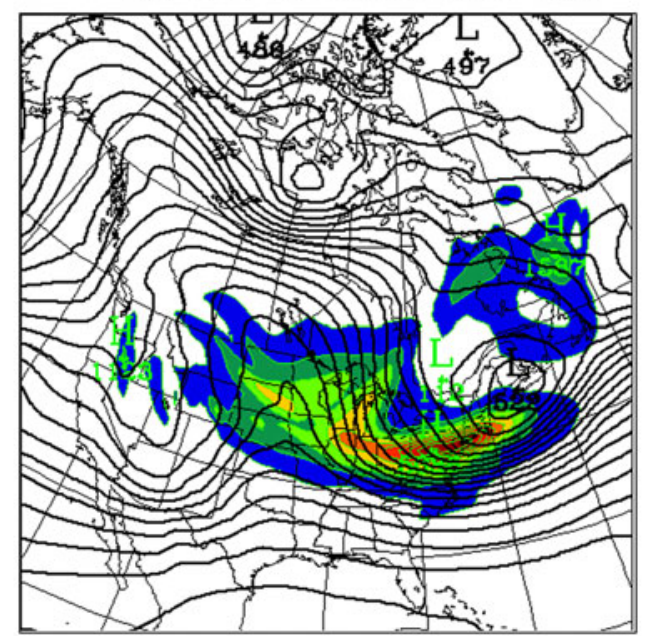

(f) 1200 UTC 6 December 1992

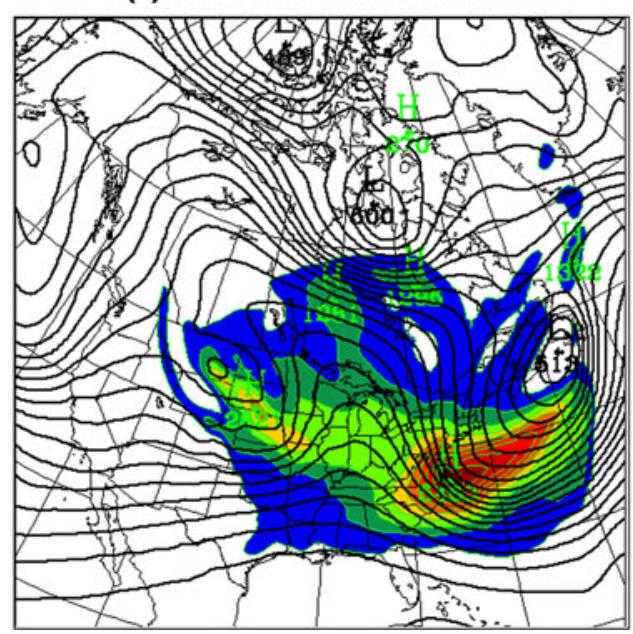

contour) is contoured at 4 dam intervals, while the CRCM-perturbation total energy is plotted in colors
5000

4500

4000

3500

3000

2500

2000

1500

1000

500

0 


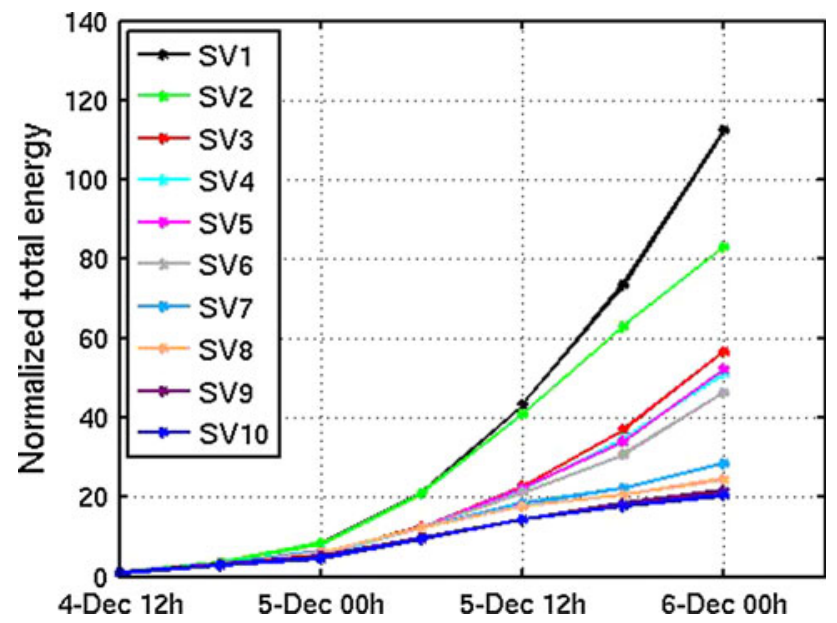

Fig. 4 Total-energy amplification for the ten SVs during the 36-h period
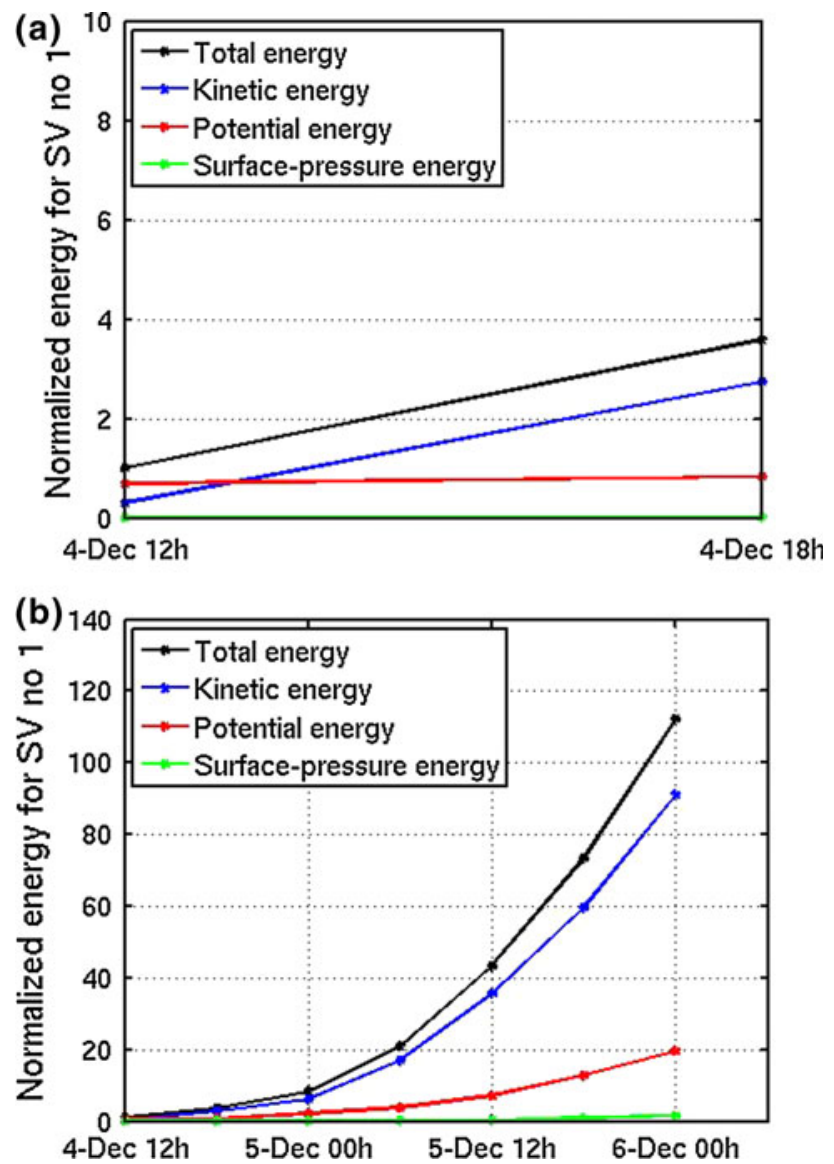

Fig. 5 36-h evolution for the leading SV energy partitioned in kinetic, potential and surface-pressure components. All terms are normalized by the initial total energy. a Shows a zoom on the initial time

Regarding the vertical structure, Figs. $7 \mathrm{~b}, 8 \mathrm{~b}$ and $9 \mathrm{~b}$ reveal at initial time a strong westward (upstream) tilt with height, especially in the mid-troposphere, the structure at (a) 4 December $12 \mathrm{~h}$

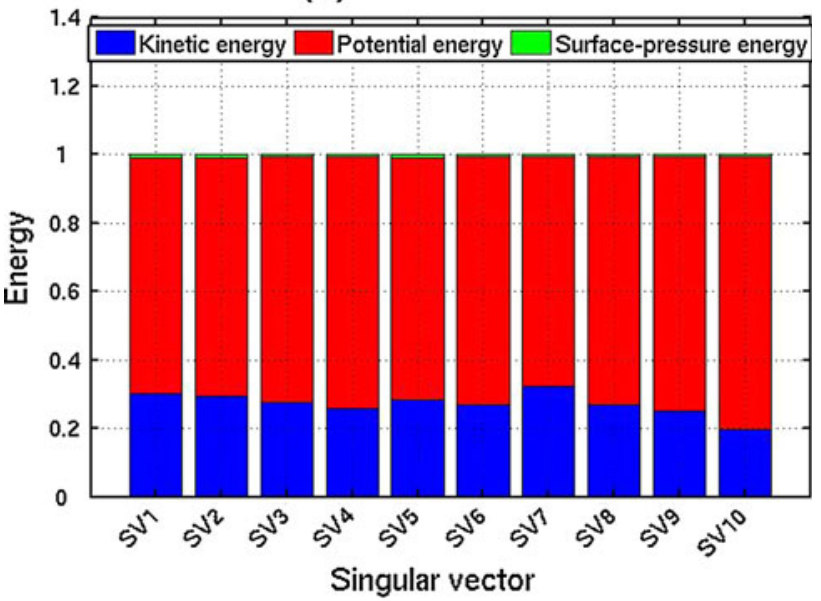

(b) 6 December 00h

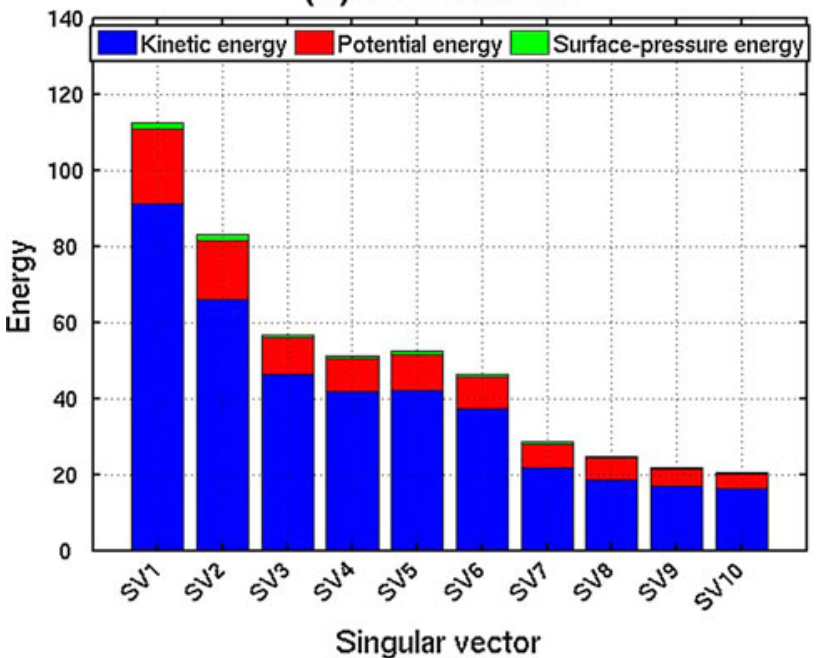

Fig. 6 SV energy partitioned in kinetic, potential and surfacepressure components at $\mathbf{a}$ initial and $\mathbf{b}$ final time normalized by the initial total energy

the upper levels being situated close to the western limit of the initial-norm domain. As can be seen in Figs. $7 d, 8 d$ and $9 \mathrm{~d}$, the westward tilt reduces as the perturbation grows and only a small tilt remains in the main pattern of the temperature and zonal-wind perturbation, which suggests that the perturbation will continue to grow but at a slower rate. As explained by Hoskins et al. (2000) and Coutinho et al. (2004), this westward tilt is a major characteristic of midlatitude baroclinically growing non-normal modes. This configuration allows the perturbation to grow by a conversion of the basic flow available potential energy into perturbation kinetic energy. The other 10 leading SVs have similar structures and evolutions (not shown).

For comparison, the structure of the perturbations in one of the $20 \mathrm{CRCM}$ members after $30 \mathrm{~h}$ is presented in Fig. 7e, $\mathrm{f}$ for the temperature field, in Fig. 8e, f for meridional wind and in Fig. 9e, f for zonal wind. Note the 
(a) SV no. 1 at initial time

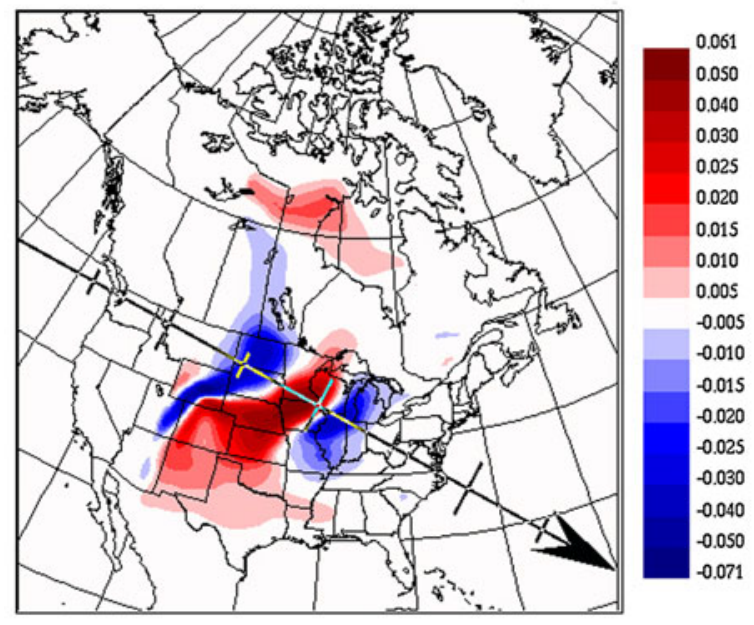

(c) SV no. 1 after 30 hours

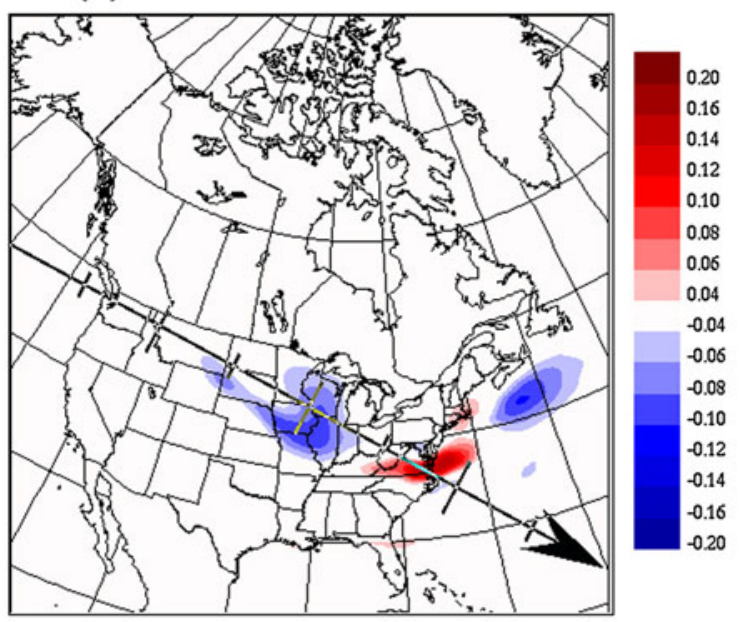

(e) NA08 perturbation after 30 hours

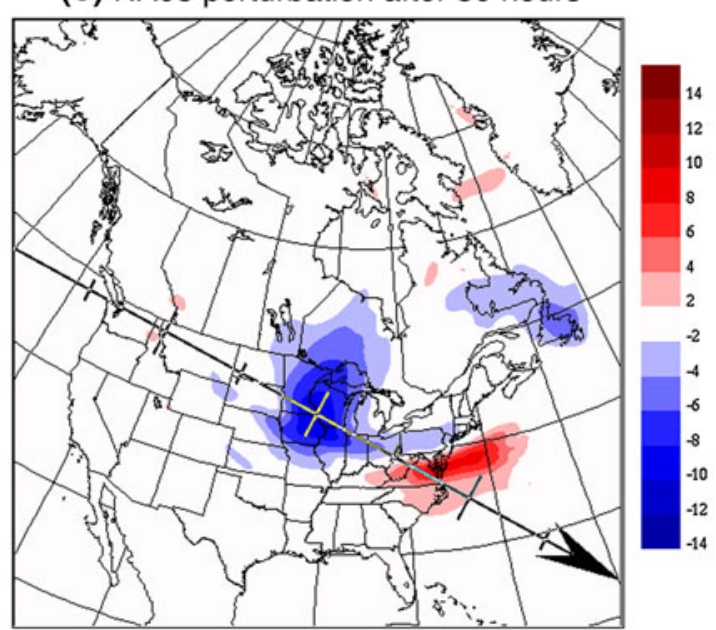

Fig. 7 Temperature (in ${ }^{\circ} \mathrm{C}$ ) horizontal structure at 0.688 eta level and vertical cross sections along the arrow corresponding to $(\mathbf{a}, \mathbf{b}, \mathbf{c}, \mathbf{d})$ the leading SV normalized by the initial total energy at (a, b) initial (b) SV no. 1 at initial time

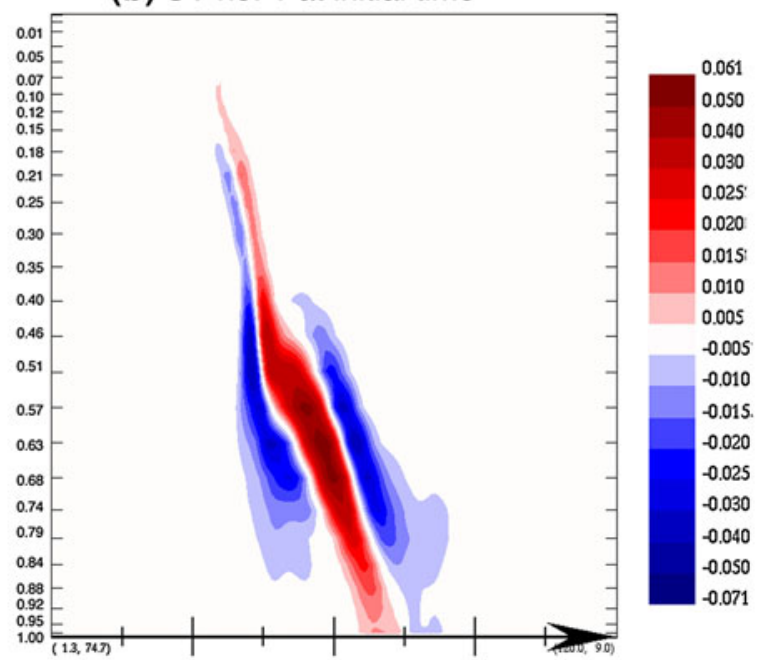

(d) SV no. 1 after 30 hours

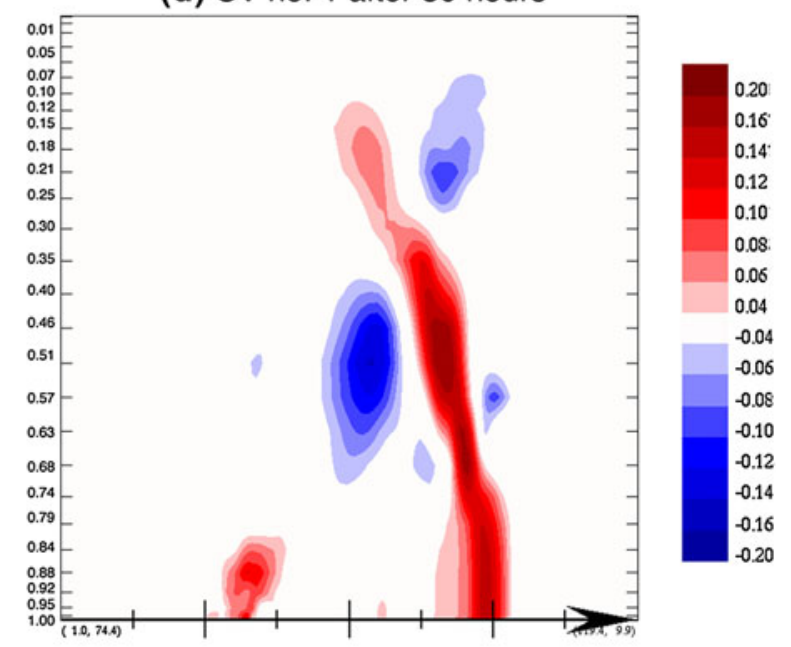

(f) NA08 perturbations after 30 hours

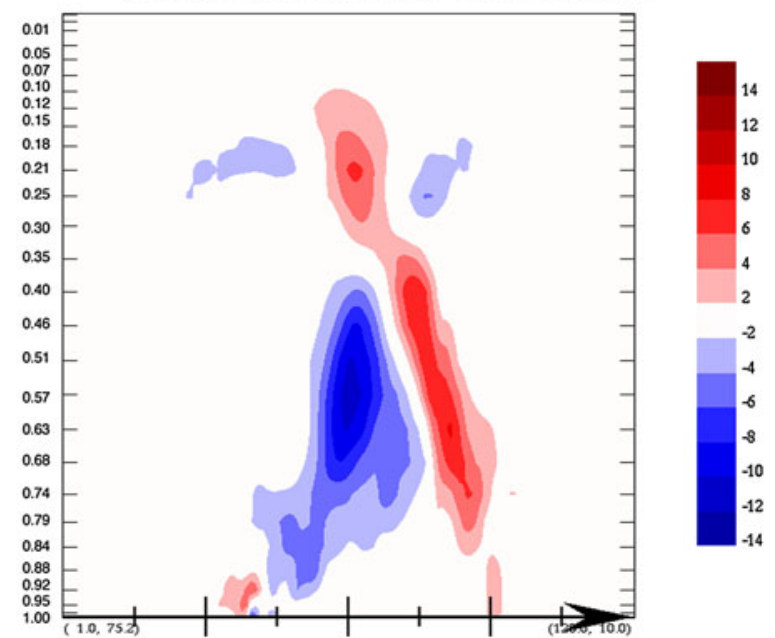

time and at (c, d) after $30 \mathrm{~h}$ and corresponding to (e, f) NA08 CRCM perturbation on 1800 UTC 5 December. To facilitate the comparison, the SV was multiplied by -1 
(a) SV no. 1 at initial time

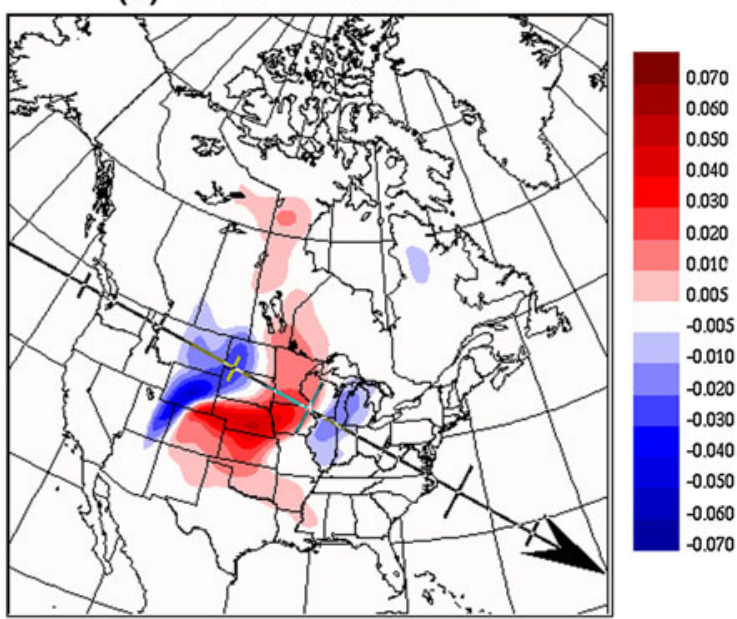

(c) SV no. 1 after 30 hours

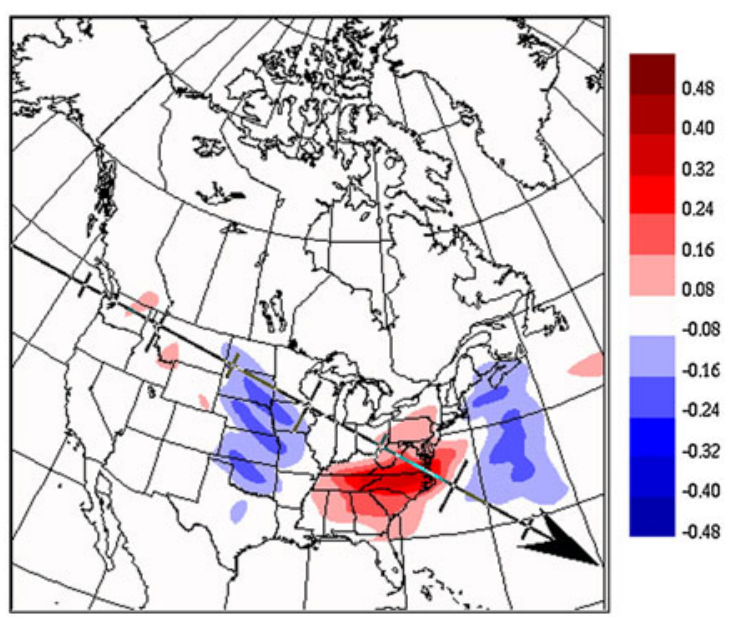

(e) NA08 perturbation after 30 hours

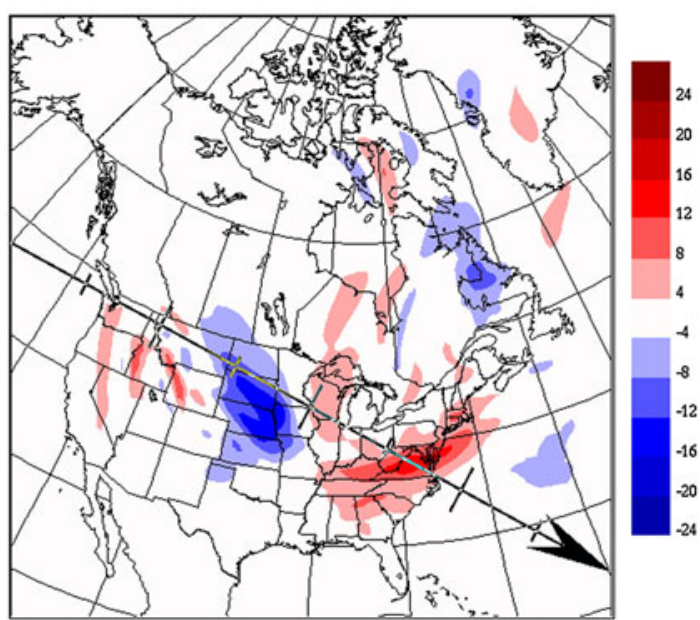

Fig. 8 Meridional wind (in $\mathrm{m} / \mathrm{s}$ ) horizontal structure at 0.688 eta level and vertical cross sections along the arrow corresponding to $(\mathbf{a}, \mathbf{b}, \mathbf{c}, \mathbf{d})$ the leading SV normalized by the initial total energy at (b) SV no. 1 at initial time

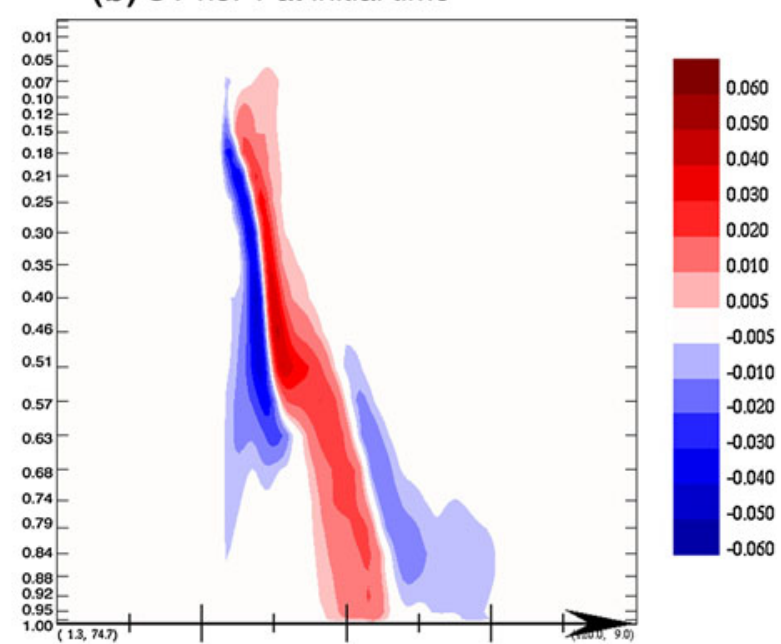

(d) SV no. 1 after 30 hours

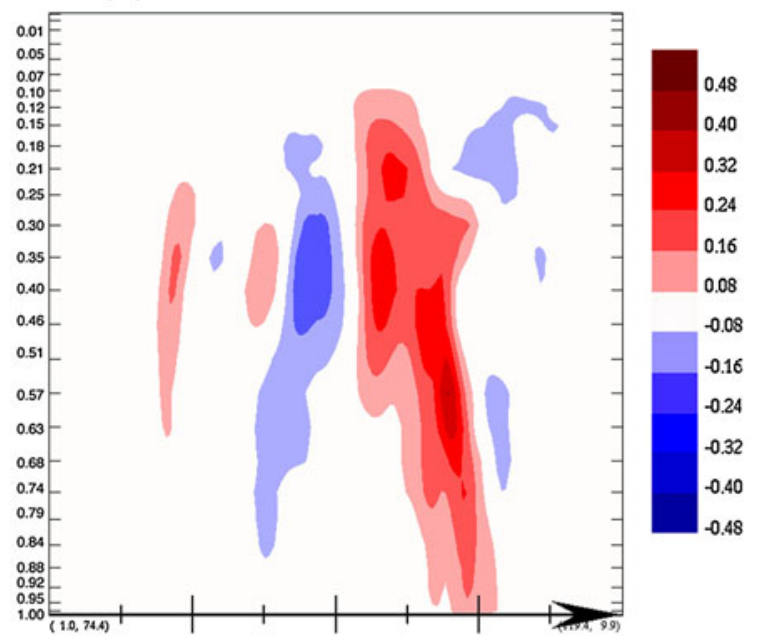

(f) NA08 perturbations after 30 hours

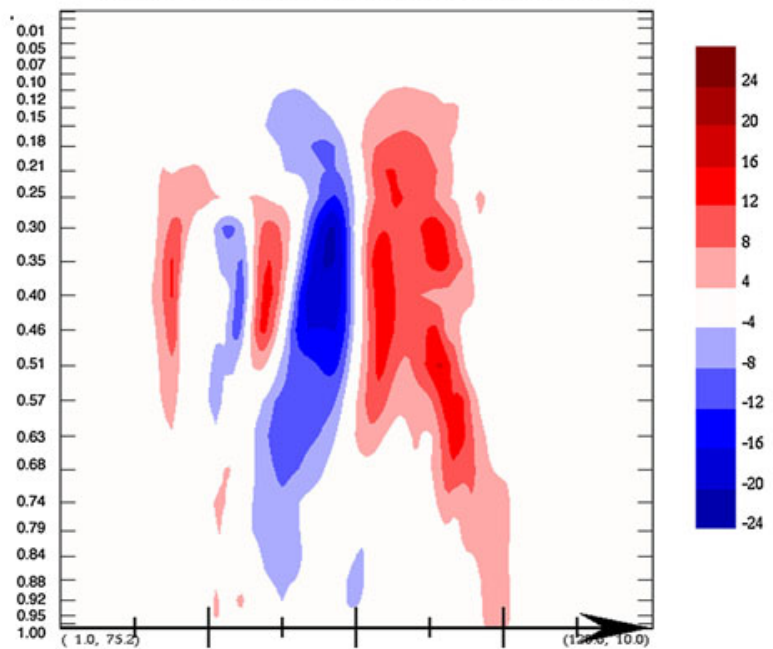

(a, b) initial time and at (c, d) after $30 \mathrm{~h}$ and corresponding to (e, f) NA08 CRCM perturbation on 1800 UTC 5 December. To facilitate the comparison, the SV was multiplied by -1 
(a) SV no. 1 at initial time

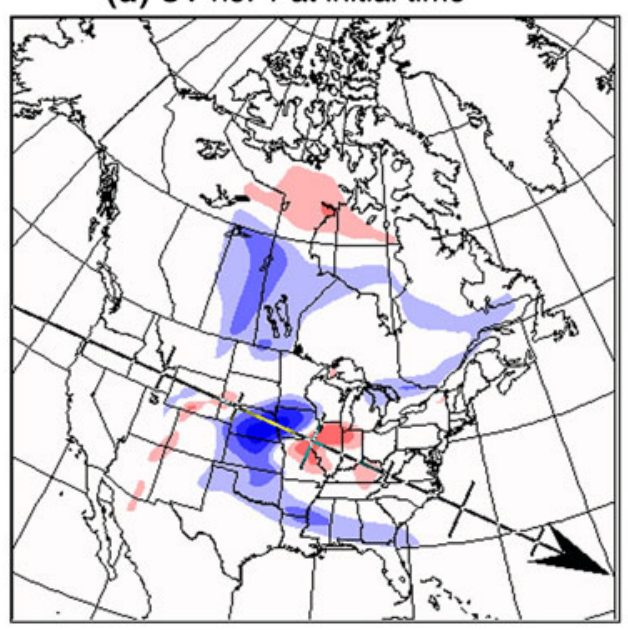

(c) SV no. 1 after 30 hours

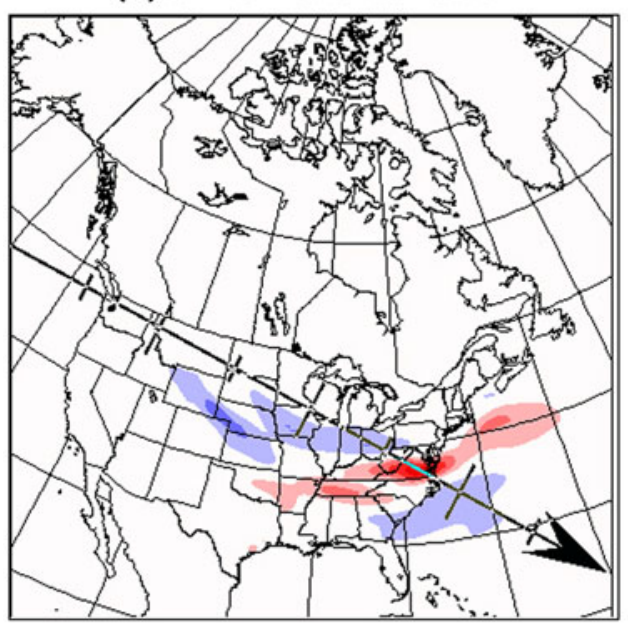

(e) NA08 perturbation after 30 hours

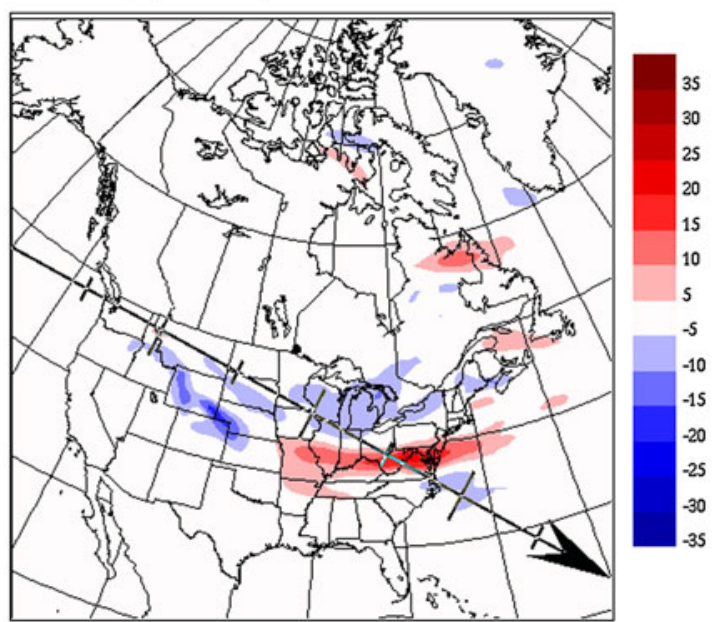

Fig. 9 Zonal wind (in $\mathrm{m} / \mathrm{s}$ ) horizontal structure at 0.688 eta level and vertical cross sections along the arrow corresponding to $(\mathbf{a}, \mathbf{b}, \mathbf{c}, \mathbf{d})$ the leading SV normalized by the initial total energy at $(\mathbf{a}, \mathbf{b})$ initial (b) SV no. 1 at initial time

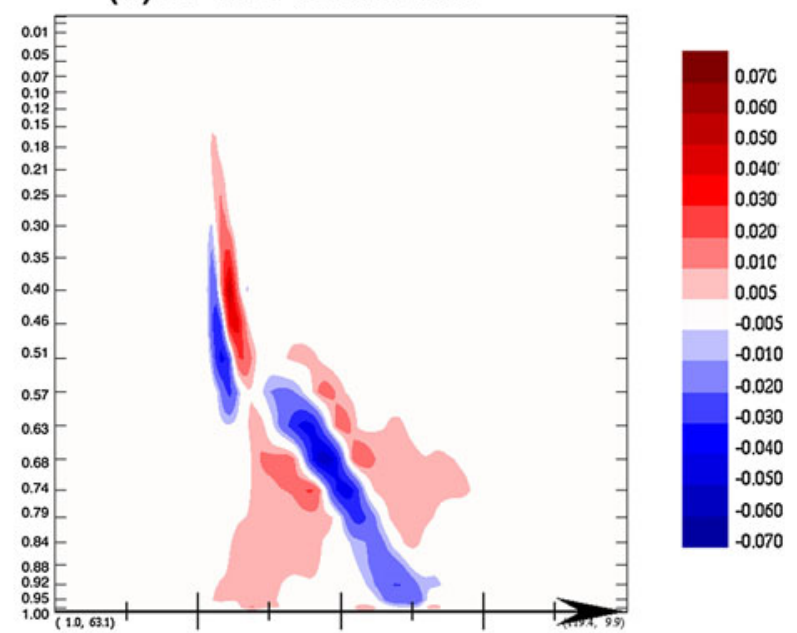

(d) SV no. 1 after 30 hours

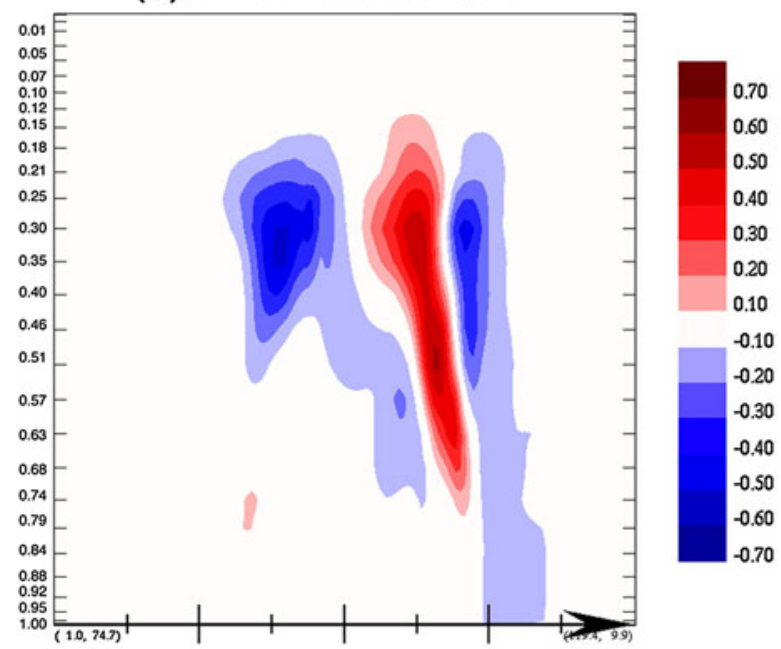

(f) NA08 perturbations after 30 hours

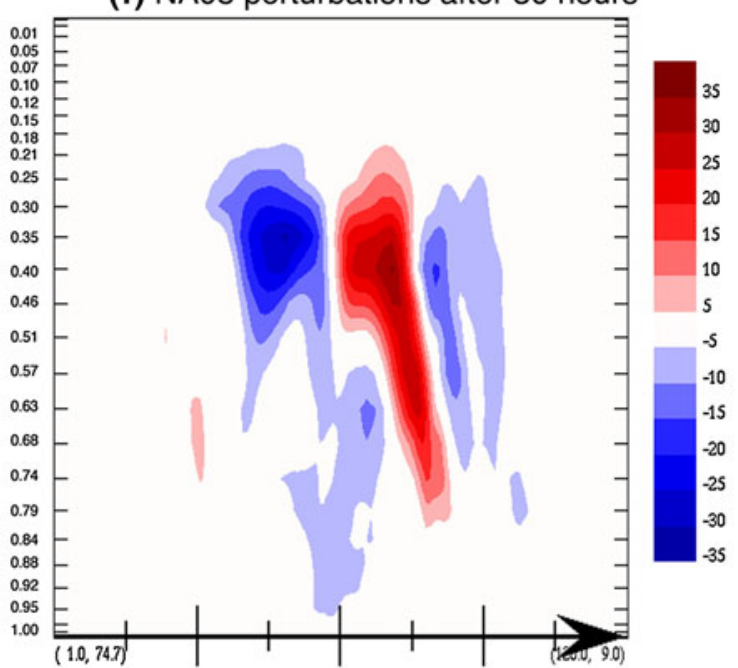

time and at (c, d) after $30 \mathrm{~h}$ and corresponding to (e, f) NA08 CRCM perturbation on 1800 UTC 5 December. To facilitate the comparison, the SV was multiplied by -1 
(a) Vertical integrated average CRCM total energy

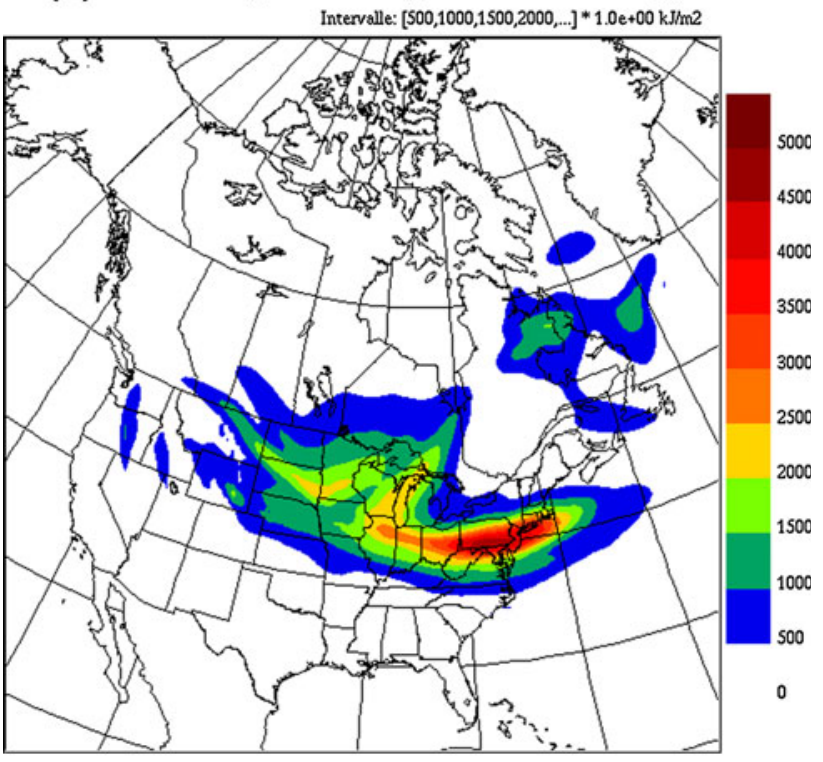

(b) SV no 1 vertical inegrated total energy

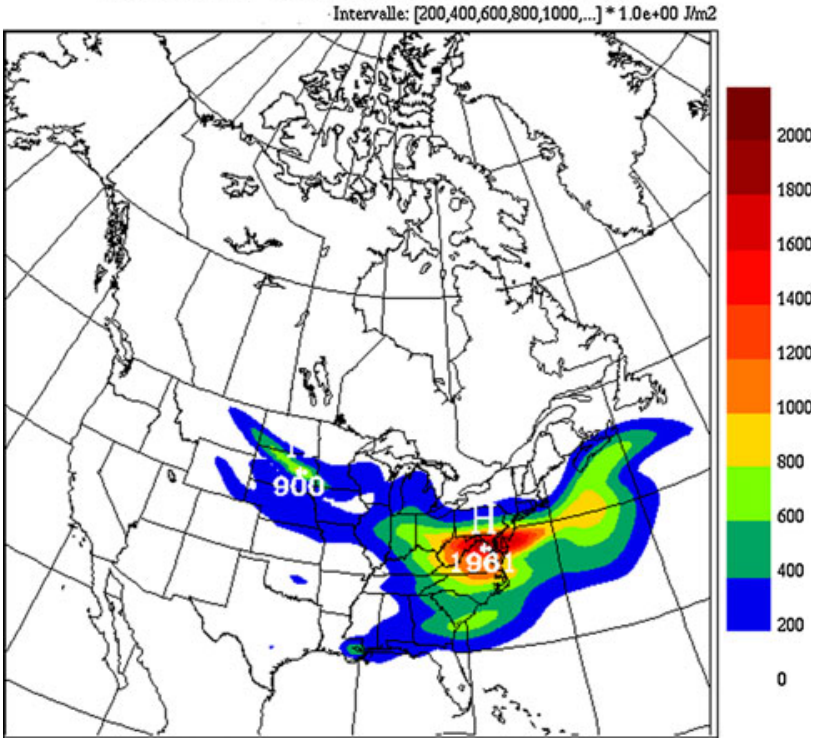

Fig. 10 Total energy horizontal distribution at 1800 UTC 5 December for $\mathbf{a}$ the CRCM perturbations and $\mathbf{b}$ first SV normalized by the initial total energy

similarity to the SV spatial patterns for all three fields, especially for the maxima situated on the continent. The temperature pattern located over the Atlantic Ocean is very weak in the CRCM case, perhaps due to the vicinity of the eastern MLB of the regional model.

We must mention that the structure of the initial SV is very different from that of CRCM perturbations (not shown) because the initial SVs are outside the attractor, pointing to areas in the system phase space where solutions do not naturally occur. However, the SVs rapidly rotate
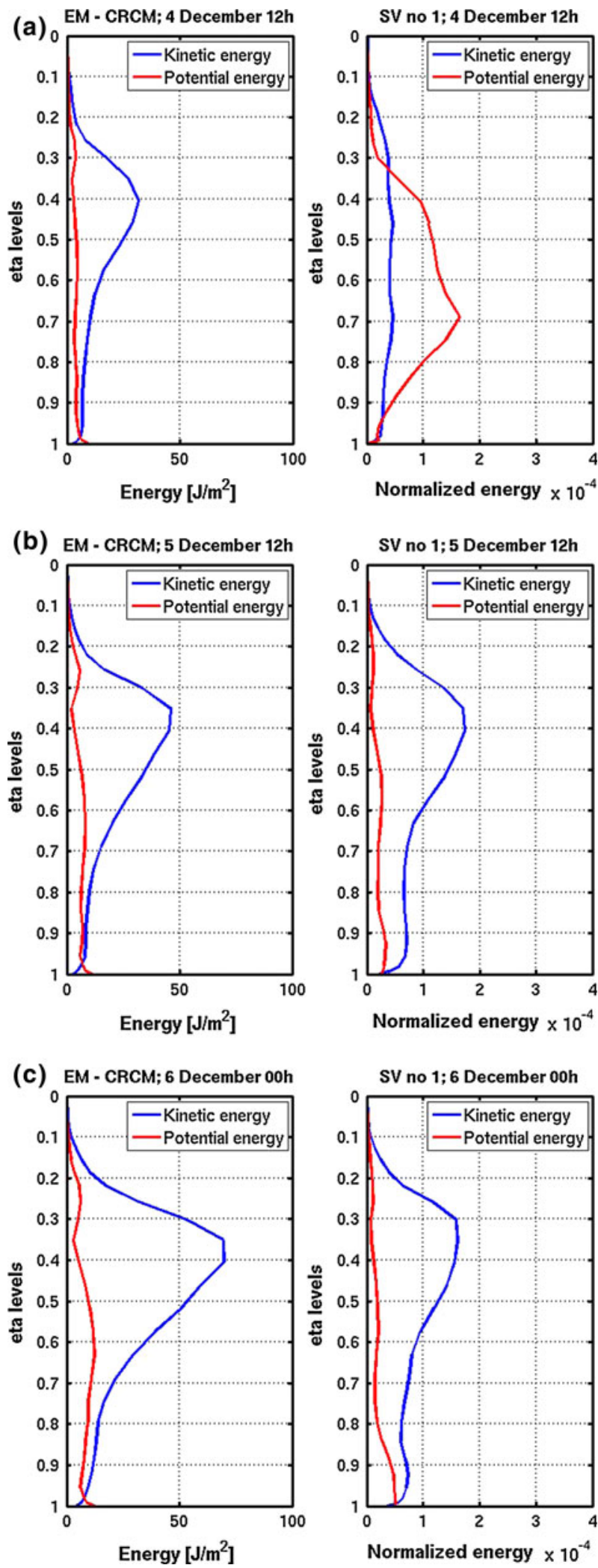

Fig. 11 Vertical distribution of (right panels) first SV kinetic and potential energy and (left panels) CRCM-perturbations average kinetic and potential energy at different moments on the $36-\mathrm{h}$ period. Note that $\mathrm{SV}$ is normalized by the total energy 

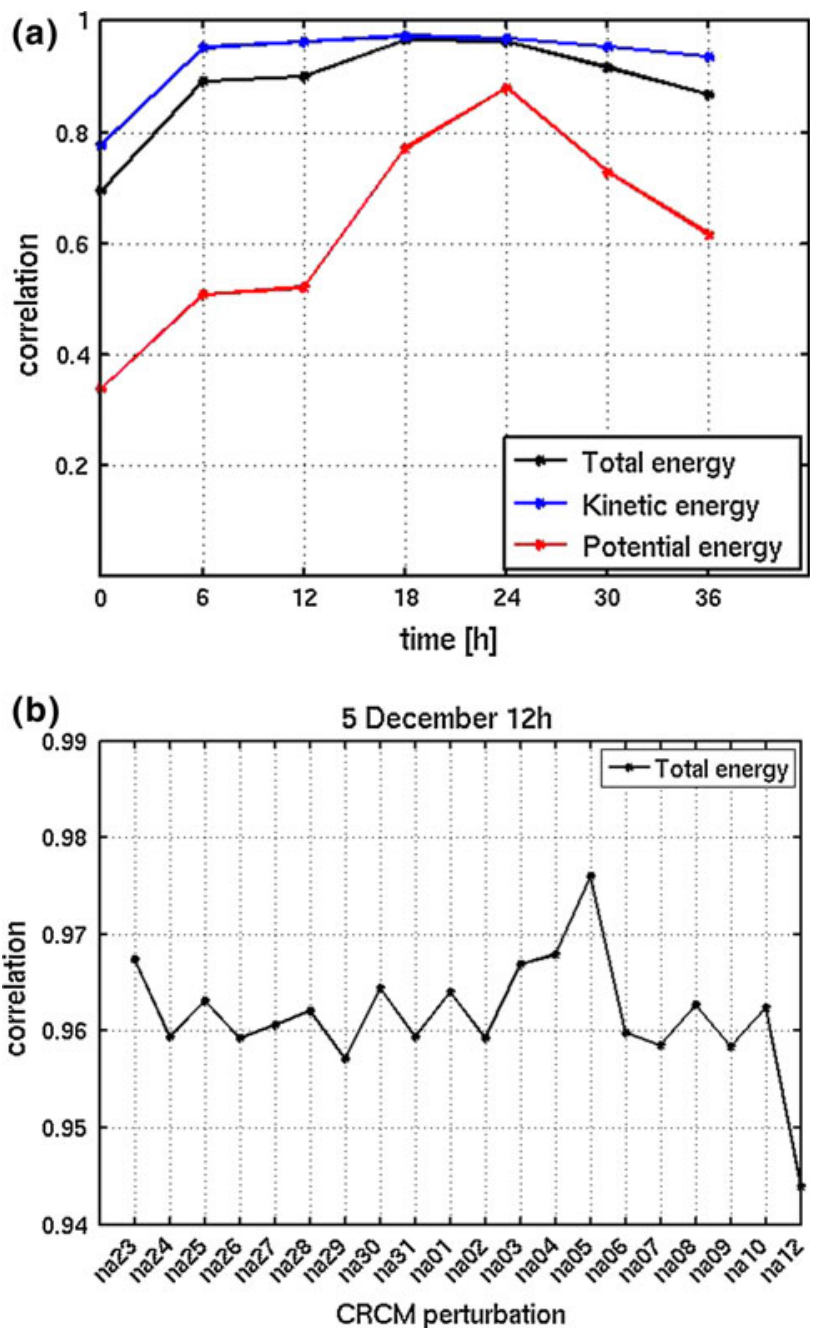

Fig. 12 a Correlation coefficient between the average vertical distributions of CRCM perturbations and first SV energy as function of time for the 36-h period. b Correlation coefficient between the vertical distributions of each CRCM perturbations and first SV total energy by 5 December $12 \mathrm{~h}$

towards the attractor and after $30 \mathrm{~h}$ the leading $\mathrm{SV}$ shape is almost identical to the CRCM evolved perturbations.

\subsubsection{Horizontal and vertical distribution of energy}

Figure 10 presents the vertically integrated total energy of the CRCM IV calculated with the 20 members on December 5 at 18:00 UTC, and for the leading SV after $30 \mathrm{~h}$. Note the collocation of the maxima and the similarity of the overall pattern. Nevertheless, the SV is slightly displaced eastward of the CRCM perturbations, while the CRCM field is more extended over the American continent.

The rapid rotation of SVs toward the CRCM solutions is also reveal by Fig. 11, which displays the vertical distributions of the horizontally integrated kinetic and potential energy of the leading SV and of the CRCM's IV, on
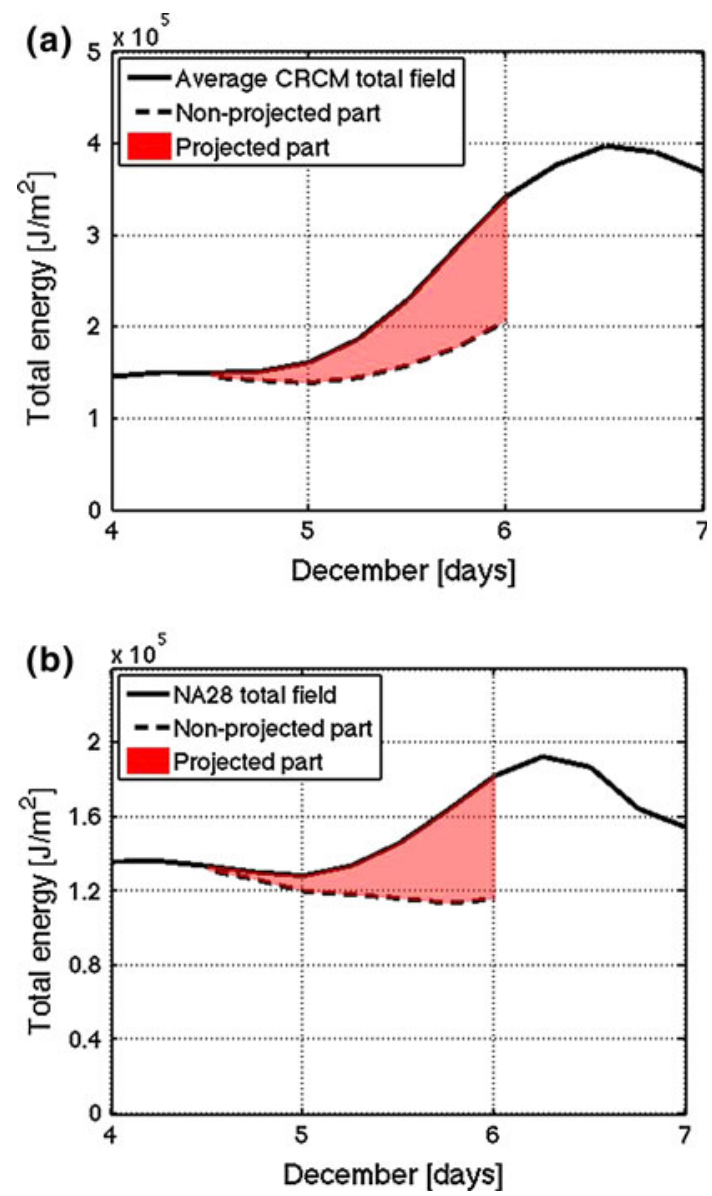

Fig. 13 a The average total energy of CRCM perturbations and $\mathbf{b}$ the total energy of NA28 perturbation: (dashed black line) non-projected part, (red area) projected part and (solid black line) total field

December 4th 12:00 UTC (initial time; top panels), $24 \mathrm{~h}$ later (middle panels), and on December 6th 00:00 UTC (low panels). Once again, the SV's amplitude has been normalised to correspond to a unit total energy $\left(1 \mathrm{Jm}^{-2}\right)$. At initial time, the potential energy of the leading SV is larger than the kinetic component and peaks around the 700-hPa level (right panel, Fig. 11a). This is quite different from the CRCM, where the kinetic energy dominates, with a maximum near $400 \mathrm{hPa}$. Twenty-four hours later, the SV kinetic energy is much greater than the potential energy, similar to CRCM perturbations, with maximum kinetic energy near $400 \mathrm{hPa}$. The similarity persists up to $36 \mathrm{~h}$. Nevertheless, some differences are noted at lower levels, close to the surface, especially in the potential energy, which has a near-surface maximum in the case of SV (this was already noticeable in Fig. 7d, f).

Correlation coefficients between the vertical distribution of CRCM IV energy and of the leading SV as a function of time are shown in Fig. 12a. For the total energy, the correlation grows from a minimum value of 0.7 at the initial time (December 4th 12:00 UTC) to 0.96 at $24 \mathrm{~h}$. At this 
time, all CRCM members perturbations have a similar high correlation coefficient with the leading SV, as can be seen in Fig. 12b. The correlations are higher when only kinetic energy is considered and much smaller for the potential energy. These small correlations in potential energy are due primarily to the surface maximum noted in the SV case. Several factors can explain the differences at lower levels. First, interpolation/resolution issues: SVs are computed on a global uniform grid with $1^{\circ}$ horizontal resolution and next interpolated on the CRCM grid, which has a $0.5^{\circ}$ horizontal resolution. Second, the accuracy of the TLM: the evolved SVs are the result of TLM propagation with simplified physics, while CRCM perturbations are the result of non-linear model integration with complete physics, and at lower levels the non-linear processes can be even more important.

It is interesting to note, however, that the overall comparison between the CRCM perturbations and the set of SVs has showed a remarkable similarity between the CRCM perturbations and the most unstable SV after $30 \mathrm{~h}$ of integration, especially in regions with maximum total energy.

\subsection{Projection on the set of singular vectors}

Let $x_{k}\left(u_{k}, v_{k}, T_{k}, p_{S k}, \ldots\right)$ represent the CRCM $k$ th member perturbation, and $y_{j}\left(u_{j}, v_{j}, T_{j}, p_{S j}, \ldots\right)$ be the $j$ th $\mathrm{SV}$. Given the orthogonality for the total energy, and if the SV are normalized (total energy equal to one), the projection of $x_{k}$ on a complete base of $N$ SVs at time $t$ is:

$x_{k}(t)=\sum_{j=1}^{N} \alpha_{k j} \hat{y}_{j}(t)$,

where $\hat{y}_{j}(t)$ represents the total-energy normalized SV and $\alpha_{k j}=\left\langle x_{k}(t), E_{t} \hat{y}_{j}(t)\right\rangle$ the projection coefficients. If a truncated (not complete) basis of ten SVs is considered, the CRCM perturbation can be written as:

$x_{k}=\sum_{j=1}^{10} \alpha_{j k} \hat{y}_{j}+\sum_{j=11}^{N} \alpha_{j k} \hat{y}_{j}=\tilde{x}_{k}+\Delta x_{k}$,

with $\tilde{x}_{k}$ the projected part into the truncated basis, and $\Delta x_{k}$ the non-projected part representing slower growing, neutral or decaying vectors.

The total energy of the projected part will be given by the sum of the squares of the projection coefficients,

$E\left(\tilde{x}_{k}\right)=\left\|\tilde{x}_{k}(t)\right\|^{2}=\sum_{j=1}^{N} \alpha_{k j}^{2}$,

and the CRCM perturbation total energy can be written as:

$E\left(x_{k}\right)=\sum_{j=1}^{10} \alpha_{j k}^{2}+\sum_{j=11}^{n} \alpha_{j k}^{2}=E\left(\tilde{x}_{k}\right)+E\left(\Delta x_{k}\right)$.
Figure 13a displays the average values of the energy corresponding to all the CRCM members (NA23 to NA12): the total energy $\left(\frac{1}{20} \sum_{k=1}^{20} E\left(x_{k}\right)\right)$ in a solid black line, and the non-projected part of total energy $\left(\frac{1}{20} \sum_{k=1}^{20} E\left(\Delta x_{k}\right)\right)$ in a dashed black line. The red area indicates the projected part of total energy $\left(\frac{1}{20} \sum_{k=1}^{20} E\left(\tilde{x}_{k}\right)\right)$, i.e. the part of CRCM perturbations' total energy represented by the ten leading SVs. The particular case of one CRCM member, $N A 28$, is displayed in Fig. 13b. Note that initially only a negligible part of the CRCM perturbations are projected into the ten SVs sustaining the idea that SVs are outside the system's attractor at initial time. As time passes, the projected part grows rapidly, and after $36 \mathrm{~h}$ it represents a significant fraction of the total CRCM perturbation growth: by 00:00 UTC 6 December, the projected part reaches the value of $1.35 \times 10^{5} \mathrm{~J} \mathrm{~m}^{-2}$, which is about $40 \%$ of the average total energy of the CRCM perturbations, or $70 \%$ of the CRCM IV growth in $36 \mathrm{~h}$, the other $30 \%$ of the CRCM growth being due to the non-projected part growth. Therefore, $70 \%$ of the CRCM-perturbations growth is explained by the first ten SVs. This represents an average over the twenty CRCM members. In the case of NA28 member (Fig. 13b), the nonprojected part diminishes in time and consequently the projected part accounts for the entire growth.

Figure $14 \mathrm{a}, \mathrm{b}$ show the $6 \mathrm{~h}$-mean growth rate of the projected and the non-projected parts, estimated as

$r_{k}(t)=\frac{1}{\Delta t} \frac{E_{k}(t)-E_{k}(t-\Delta t)}{E_{k}(t-\Delta t)}$,

with $\Delta t=6 \mathrm{~h}$, and expressed in units of percent per hour. Figure 14a represents the perturbations of all CRCM members (shaded area) and their mean value (the solid line) while Fig. 14b represents the case of one CRCM member perturbation (NA28). During the first hours, the projected part (the part represented by the first ten SVs) is made of small-scale small-amplitude perturbations with large growth rates of $80-170 \%$ per hour, while the nonprojected part is composed of large-scale large-amplitude perturbations with very small growth or even decaying rates as in the case of NA28 perturbation (Fig. 14b). As time passes, the projected part grows in spatial scale and gains in amplitude, but its growth rate decreases, approaching the rate of the non-projected part.

The rotation of leading SV toward the CRCM solutions is also evident in Fig. 15, which shows the meridional wind near $460 \mathrm{hPa}$ of the NAO8 perturbation projected on the leading SV (Fig. 15a, b) and of the NAO8 total perturbation (Fig. 15c, d) at initial time and after $30 \mathrm{~h}$. The difference between Fig. 15a, c illustrates the relatively large scales aspect of the non-projected part at the initial time and the very small projection of the CRCM perturbation on the initial SV. 
(a)
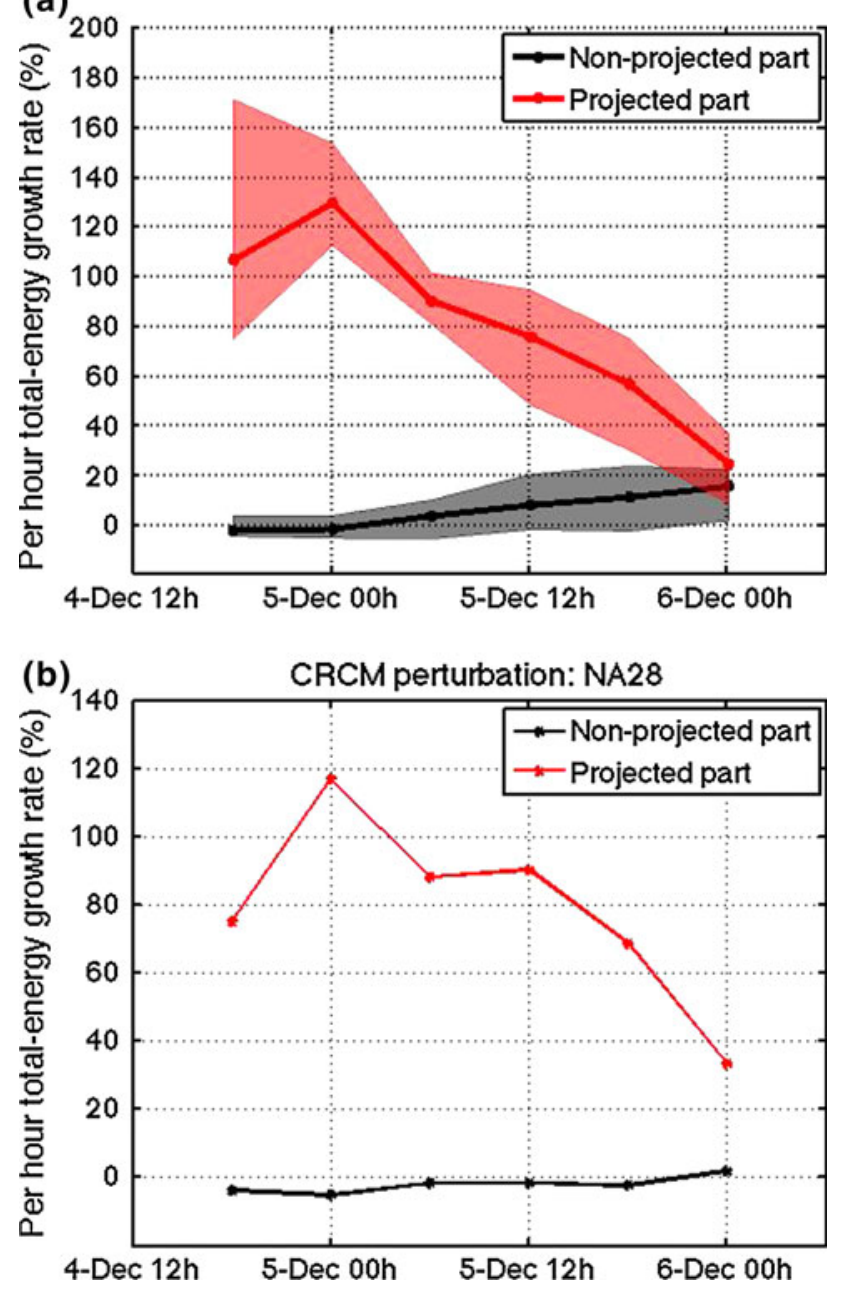

Fig. 146 h-mean growth rate in percentage per hour for a all CRCM-perturbations and b NA28 perturbation total-energy (red line) projected part and (black line) non-projected part

As mentioned previously, the first leading SV distinguishes itself from the other SVs by its high amplification rate. An important question that arises from this is whether there is a preference of the projection of CRCM IV on this particular SV. Figure 16 shows fraction of CRCM perturbations energy explained by each of the ten SVs at 18:00 UTC on December 5th and confirms the perturbations project significantly on the first leading SV. The first leading SV grows rapidly enough to account in average for over $23 \%$ of the CRCM total perturbation energy and for more than $50 \%$ of the projected part. Note that a particular member, $N A 12$, has a very small projection. This is due to the fact that the NA12 is very close to the reference simulation; as a consequence, the perturbation energy of this particular member is very small, its total energy being about eight times smaller than the average total perturbation energy of other members in the ensemble.
While, Fig. 16 presents the fraction of each of the CRCM-perturbation energy explained by each of the ten SVs, Fig. 17 shows the average value (ensemble mean) of the CRCM projected part on each of the ten SVs, from the initial time to the final time. The figure reveals that the perturbation is predominantly projected on the most unstable SV through most of the growth period. At final time, the projections on each of the other nine SV are small compared to the projection on the leading SV. However, when adding them together, they have an important contribution to the total projected part.

The set of SVs used in this study contains only the first ten SVs. Studies that used more than ten SVs (e.g., Errico et al. 2001; Snyder and Hakim 2005) have shown that if the number of amplifying SVs is very large and the spectrum of growth rates is flat, then the probability that a random perturbation projects strongly on any single or small set of SVs is correspondingly small. Even though subsequent growth in the leading SVs will be greater than growth in other individual SV, the overall behaviour can be dominated by the much larger set of non-leading SVs.

\section{Summary and concluding remarks}

This paper focused on the physical understanding of specific episodes with rapid growth of IV in Canadian Regional Climate Model (CRCM) simulations. It was hypothesized that periods of important IV growth might arise from local dynamical instabilities. Initially small perturbations in unstable regions begin to develop while they are advected by the mean flow toward the lateral boundaries where they are eventually transported out of the domain.

A possible way to approach the question of hydrodynamical instabilities is through the singular vector (SV) approach. Hence, our focus was on comparing the CRCM perturbations with a set of SVs and ascertaining whether the IV growth may be linked to the most unstable SV.

To test the hypothesis, a 21-member CRCM ensemble of simulations was performed and the analysis was focused on one specific episode of large IV growth occurring between 4 and 6 December 1992. The ensemble was decomposed into a reference simulation and a set of perturbations with respect to that reference. Then, the total energy of each CRCM perturbation was computed, equivalent to the dry total-energy norm used to compute the set of SVs. The set of SVs was restricted to the leading ten, archived at every $6 \mathrm{~h}$, using an optimization time interval (OTI) of $36 \mathrm{~h}$, and initial- and final-time norms restricted to a sub-domain contained within the CRCM integration area. We found that the ten leading SVs were all growing perturbations, the first SV dominating the others. 
(a) 12:00 UTC; 4 December 1992

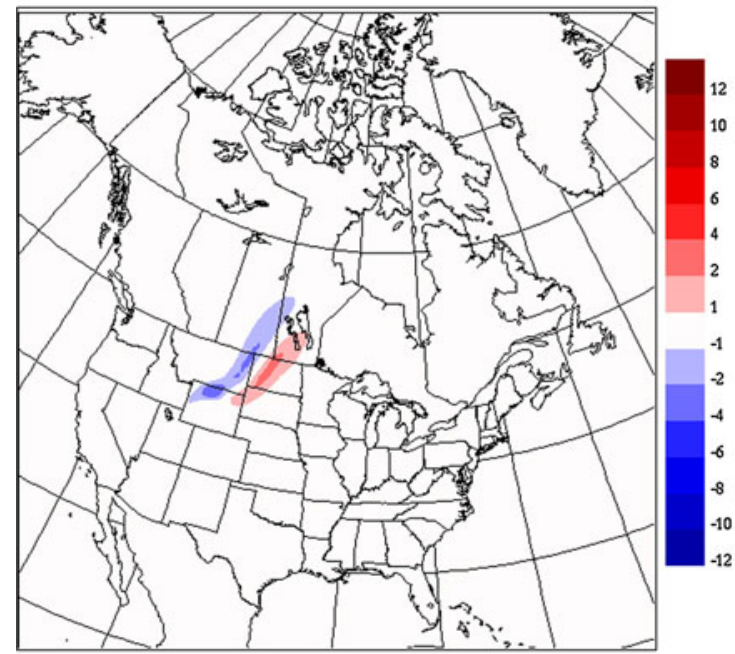

(c) 12:00 UTC; 4 December 1992

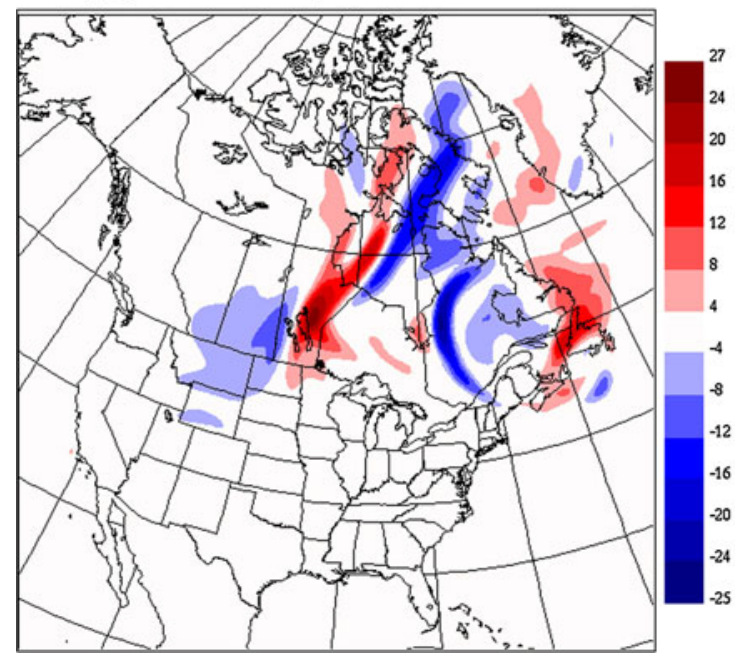

(b) 18:00 UTC; 5 December 1992

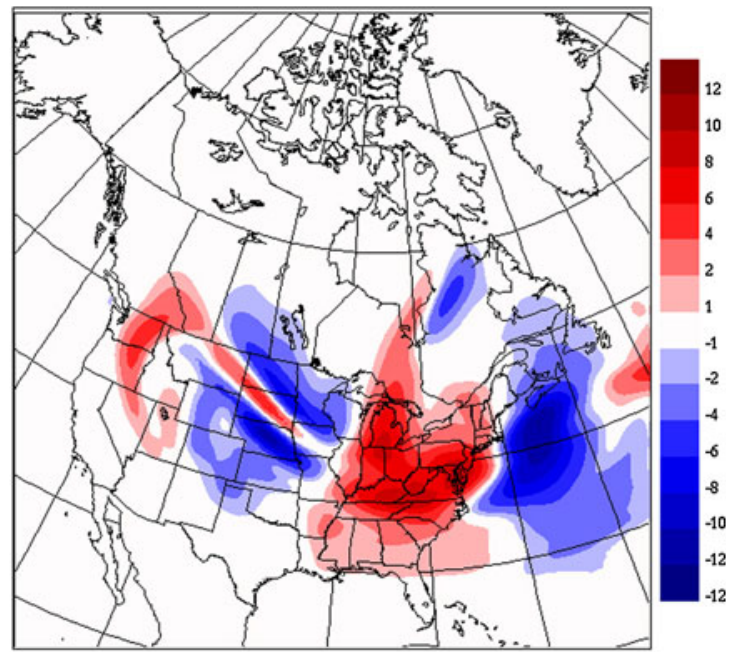

(d) 18:00 UTC; 5 December 1992

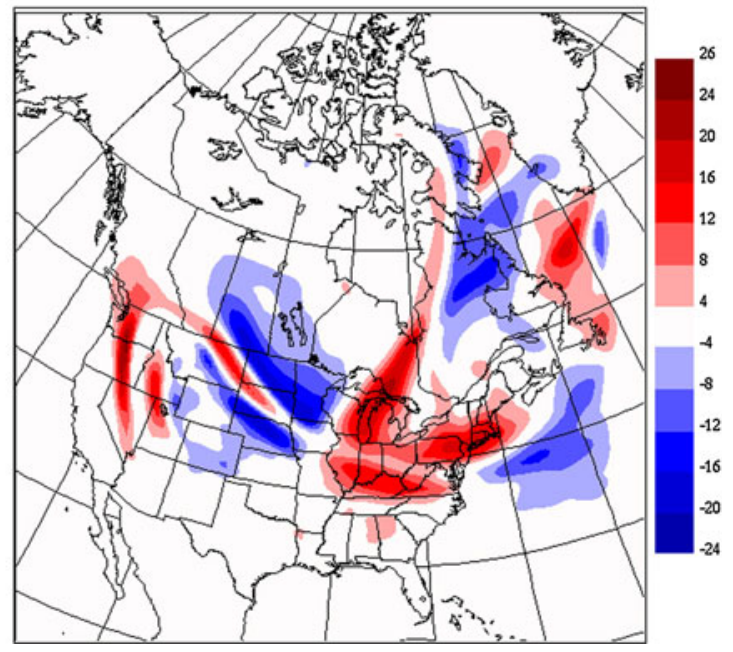

Fig. 15 Meridional wind (in m/s) at approximately $460 \mathrm{hPa}$ for (a, b) NA08 projected part on the first SV and (c, d) NA08 total field at initial time and after $30 \mathrm{~h}$

The comparison was first focused on SV properties such as perturbation structure, temporal evolution of energy, energy partition and spatial distribution. A remarkable structural similarity was found between the CRCM perturbations and the leading SV after 24- to 36-h tangent-linear model integration. The spatial patterns were roughly the same, presenting a westward (upstream) tilt in the temperature and zonal wind fields. It was also shown that all ten SVs present at final time a similar partition of energy, with dominant kinetic energy component, as for the CRCM perturbations. The overall agreement was illustrated by comparing the horizontal and vertical distribution of the first SV's energy with the horizontal and vertical distribution for the average of the energy of the CRCM perturbations. The comparison revealed similar overall patterns and a collocation of total-energy maxima after 24-36 h.
We have also computed the projection of the CRCM perturbations on the truncated base of ten leading SVs. It was shown that only a very small part of the CRCM perturbations initially projected on the ten SVs. During the next $36 \mathrm{~h}$, the projected part grew very much, and ended up representing an important part of the total CRCM perturbation growth after $36 \mathrm{~h}$. Quantitatively, up to $40 \%$ of the average CRCM total energy was projected on the ten SVs at final time, the first SV accounting in average for over $23 \%$ and the rest being projected into the nine remaining SVs, for which only small projection amplitudes did occur. Other slowly growing or decaying perturbations represented the non-projected part. As a consequence, the overall picture showed that even though subsequent growth in the leading SV was greater than growth in other individual SVs, the total energy was dominated by the much 
Fig. 16 Fraction of CRCMperturbations total energy explained by each SV by 1800 UTC 5 December

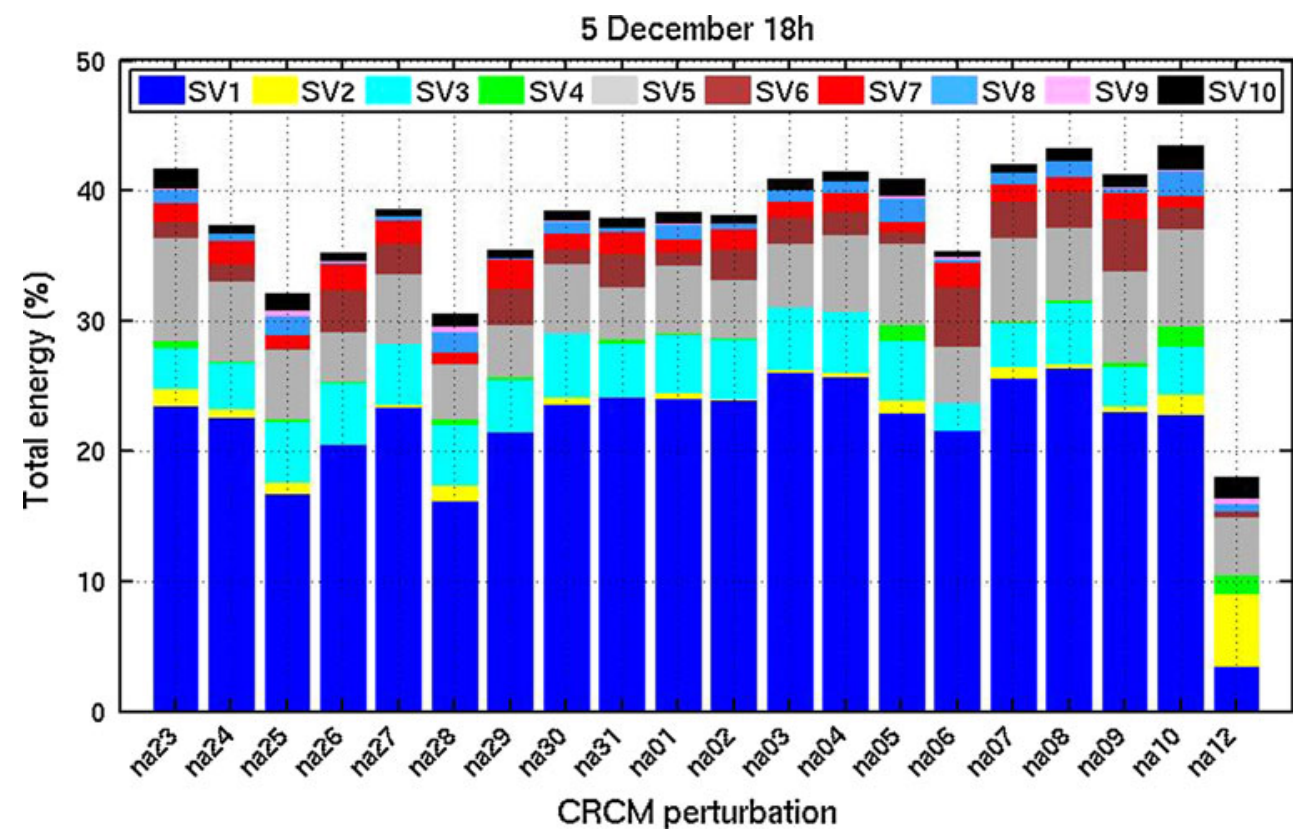

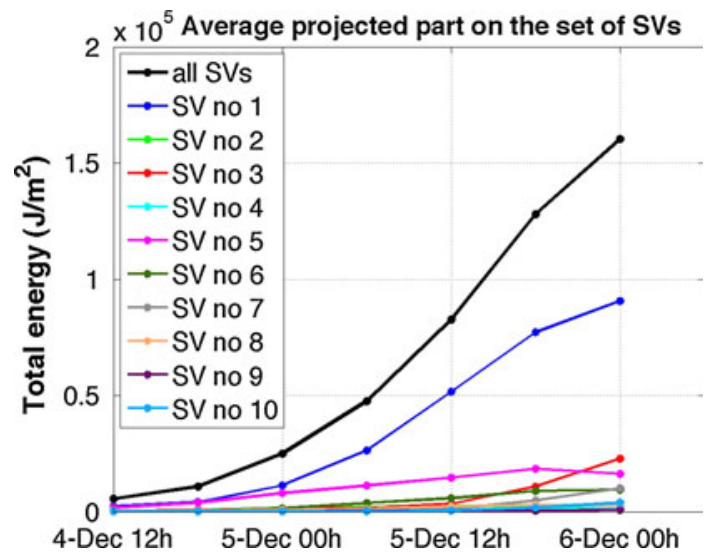

Fig. 17 Average total-energy temporal evolution for the CRCMperturbations projected part on each SV

larger set of non-leading SVs from initial to final time. This behaviour is in accord with the previous results of Errico et al. (2001) and Snyder and Hakim (2005) who used more than ten SVs in their analysis of four different synoptic cases, and cyclogenetic perturbations in the context of the quasi-geostrophic Eady model, respectively.

Despite the fact that the total energy was dominated by the slow-developing non-leading SVs, the growth itself was explained in a large proportion by the most rapid SV, which accounted in average for over $50 \%$ of the CRCM-perturbations growth in $36 \mathrm{~h}$. These results suggest that the growth of CRCM IV was due to the growth of unstable perturbations, the most part being represented by the growth of the first leading SV from the linear operator. The results showed a high similarity between the CRCM perturbations and the first SV after 24- to 36-h tangent-linear model integration. This SV had initially a predominantly low- to mid-level, westward tilted structure, which was followed by a vertical alignment and amplitude amplification.

The success of the SV analysis is probably due to the fact that, unlike the IV in an ensemble of Global Climate Model simulations, the RCM IV is subject to lateral boundary conditions constraint. As a consequence, the differences between the members in an RCM ensemble behave most of the time as small perturbations with rapid growth and therefore can be decomposed in terms of SVs.

In conclusion, we found that final-time SVs are useful to explain growth of IV in regional climate simulations. It was demonstrated that the projection on the first SV at final time is greater than the projection on other non-leading SVs and there is a high similarity between the CRCM perturbations and the first SV after 24- to 36-h tangentlinear model integration. However, the initial perturbations do not project well on the initial SVs sustaining the idea that the SVs are outside the attractor of the system at initial time, pointing to areas where solutions do not naturally occur.

We are aware that the results presented above are based on a single winter case and that other cases of large IV should be investigated using the same methodology to confirm our conclusions. Also, other seasons should be considered, such as the summer, although a TLM with a more complete physics-i.e. including simplified parametrizations of moist processes-would probably be necessary, as well as a norm that takes into account the humidity perturbations.

One technical limitation of this study was that we had to use a global model to compute the SVs. To focus on the 
region of interest, the CRCM domain, we simply restricted the domain of the final-time norm. Ideally, we would have used an SV calculation based on a limited-area model, which would take into account effects of the driving boundary conditions on the growing disturbances of the CRCM.

Acknowledgments This work is part of the $\mathrm{PhD}$ thesis of the first author in Environmental Sciences at Université du Québec at Montréal and supported by Natural Sciences and Engineering Research Council of Canada (NSERC) Graduate Scholarship (CGSD3), as well as by the CRCMD Network supported by the Canadian Foundation for Climate and Atmospheric Sciences (CFCAS) and the Ouranos Consortium. Ouranos and the ESCER Centre provided the computing resources. We thank Dr. Eugenia Kalnay for her comments on a previous version of the paper and the reviewer for his/her comments that helped improve the manuscript. The first author would like to thank many people for providing the technical support, in particular Michel Giguère, Georges Huard, Nadjet Labassi, Mourad Labassi and Katja Winger.

Open Access This article is distributed under the terms of the Creative Commons Attribution Noncommercial License which permits any noncommercial use, distribution, and reproduction in any medium, provided the original author(s) and source are credited.

\section{References}

Alexandru A, de Elía R, Laprise R (2007) Internal variability in regional climate downscaling at the seasonal scale. Mon Weather Rev 135(9):3221-3238

Alexandru A, De Elía R, Laprise L, Šeparović L, Biner S (2009) Sensitivity study of Regional Climate Model simulations to large-scale nudging parameters. Mon Weather Rev 137(5):16661686

Barkmeijer J, Buizza R, Palmer TN, Puri K, Mahfouf J-F (2001) Tropical singular vectors computed with linearized diabatic physics. Q J R Meteorol Soc 127:685-708

Bélair S, Crevier L-P, Mailhot J, Bilodeau B, Delage Y (2003) Operational implementation of the ISBA land surface scheme in the Canadian regional weather forecast model. Part I: warm season results. J Hydrometeorol 4:352-370

Bélair S, Mailhot J, Girard C, Vaillancourt P (2005) Boundary layer and shallow cumulus clouds in a medium-range forecast of a large-scale weather system. Mon Weather Rev 133:1938-1959

Borges MD, Hartmann DL (1992) Barotropic instability and optimal perturbations of observed nonzonal flow. J Atmos Sci 49:335-354

Buehner M, Zadra A (2005) Impact of flow-dependent analysis-error covariance norms on extratropical singular vectors. Q J R Meteorol Soc 131:1-999

Buizza R, Montani A (1999) Targeting observations using singular vectors. J Atmos Sci 56:2965-2985

Caya D, Biner S (2004) Internal variability of RCM simulations over an annual cycle. Clim Dyn 22:33-46

Christensen OB, Gaertner MA, Prego JA, Polcher J (2001) IV of regional climate models. Clim Dyn 17:875-887

Côté J, Gravel S, Méthot A, Patoine A, Roch M, Staniforth A (1998) The operational CMC-MRB global environmental multiscale (GEM) model. Part I: design considerations and formulation. Mon Weather Rev 126:1373-1395
Coutinho MM, Hoskins BJ, Buizza R (2004) The influence of physical processes on extratropical singular vectors. J Atmos Sci 61:195-209

Davies HC (1976) A lateral boundary formulation for multi-level prediction models. Q J R Meteorol Soc 102:405-418

Descamps L, Talagrand O (2007) On some aspects of the definition of initial conditions for ensemble prediction. Mon Weather Rev 135:3260-3272

Ehrendorfer M, Errico RM (1995) Mesoscale predictability and the spectrum of optimal perturbations. J Atmos Sci 52:3475-3500

Errico RM, Ehrendorfer M, Raeder KD (2001) The Spectra of singular values in a regional model. Tellus 53A:317-332

Gauthier P, Tanguay M, Laroche S, Pellerin S, Morneau J (2007) Extension of 3D-Var to 4D-Var: implementation of 4D-Var at the Meteorological Service of Canada. Mon Weather Rev 135:2339-2354

Giorgi F, Bi X (2000) A study of IV of regional climate model. J Geophys Res 105:29503-29521

Gleckler P (1996) AMIP II guidelines. AMIP Newsletter, No. 8, PCMDI/LLNL. http://www.pcmdi.llnl.gov/projects/amip/NEWS

Hartmann DL, Buizza R, Palmer TN (1995) Singular vectors: the effect of spatial scale on linear growth of disturbances. J Atmos Sci 52:3885-3894

Hoskins BJ, Buizza R, Badger J (2000) The nature of singular growth and structure. Q J R Meteorol Soc 126:1565-1580

Kain JS, Fritsch JM (1990) A one-dimensional entraining/detraining plume model and application in convective parameterization. J Atmos Sci 47:2784-2802

Kalnay E (2002) Atmospheric modeling, data assimilation and predictability. Cambridge University Press, Cambridge

Kuo HL (1965) On formation and intensification of tropical cyclones through latent heat release by cumulus convection. J Atmos Sci 22:4063

Laprise R (1992) The Euler equations of motion with hydrostatic pressure as an independent variable. Mon Weather Rev 120:197-207

Laprise R, de Elía R, Caya D, Biner S, Lucas-Picher P, Diaconescu EP, Leduc M, Alexandru A, Šeparović L (2008) Challenging some tenets of Regional Climate Modelling. Meteorol Atmos Phys (Special Issue on Regional Climate Studies) 100:3-22

Li J, Barker HW (2005) A radiation algorithm with correlated-k distribution. Part I: local thermal equilibrium. J Atmos Sci 62:286-309

Lorenz EN (1963) Deterministic non-periodic flow. J Atmos Sci 20:30-141

Lorenz EN (1965) A study of the predictability of a 28-variable atmospheric model. Tellus 17:321-333

Lucas-Picher P, Caya D, de Elia R, Laprise R (2008a) Investigation of regional climate models' internal variability with a ten-member ensemble of ten-year simulations over a large domain. Clim Dyn 31:927-940

Lucas-Picher P, Caya D, Biner S, Laprise R (2008b) Quantification of the lateral boundary forcing of a regional climate model using an ageing tracer. Mon Weather Rev 136:4980-4996

McFarlane NA (1987) The effect of orographically excited gravitywave drag on the circulation of the lower stratosphere and troposphere. J Atmos Sci 44:1175-1800

Molteni F, Buizza R, Palmer TN, Petroliagis T (1996) The ECMWF ensemble prediction system: methodology and validation. Q J R Meteorol Soc 122:73-119

Nikiema O, Laprise R (2010) Diagnostic budget study of the internal variability in ensemble simulations of the Canadian RCM. Clim Dyn 36:2313-2337

Palmer TN, Buizza R, Molteni F, Chen Y-Q, Corti S (1994) Singular vectors and the predictability of weather and climate. Phil Trans R Soc 348:459-475 
Pellerin G, Lefaivre L, Houtekamer P, Girard C (2003) Increasing the horizontal resolution of ensemble forecasts at CMC. Nonlinear Process Geophys 10:463-468

Rapaić M, Leduc M, Laprise R (2010) Evaluation of the internal variability and estimation of the downscaling ability of the Canadian regional climate model for different domain sizes over the north atlantic region using the big-brother experimental approach. Clim Dyn 36:1979-2001

Rinke A, Dethloff K (2000) On the sensitivity of a regional Arctic climate model to initial and boundary conditions. Clim Res 14:101-113

Rinke A, Marbaix P, Dethloff K (2004) IV in Arctic regional climate simulations: case study for the Sheba year. Clim Res 27:197209

Šeparović L, de Elía R, Laprise R (2008) Reproducible and irreproducible components in ensemble simulations with a regional climate model. Mon Weather Rev 136:4942-4961

Snyder C, Hakim GJ (2005) Cyclogenetic perturbations and analysis errors decomposed into singular vectors. J Atmos Sci 62:22342247

Sundqvist H, Berge E, Kristjansson JE (1989) Condensation and cloud parameterization studies with a mesoscale numerical weather prediction model. Mon Weather Rev 117:1641-1657

Szunyogh I, Kalnay E, Toth Z (1997) A comparison of Lyapunov and optimal vectors in a low-resolution GCM. Tellus 49A:200-227

Toth Z, Kalnay E (1997) Ensemble forecasting at NCEP and the breeding method. Mon Weather Rev 125:3297-3319
Uppala SM, Ilberg PW, Simmons AJ, Andrae U, Da Costa Bechtold V (2005) The ERA-40 reanalysis. Q J R Meteorol Soc 131:2961-3012

Vanvyve E, Hall N, Messager C, Leroux S, van Ypersele J-P (2007) Internal variability in a regional model over West Africa. Clim Dyn 30:191-202

Vukicevic T, Paegle J (1989) The influence of one-way interacting lateral boundary conditions on predictability of flow in bounded numerical models. Mon Weather Rev 117:340-350

Wu CC, Chen JH, Majumdar SJ, Peng MS, Reynolds CA, Aberson SD, Buizza R, Yamaguchi M, Chen SG, Nakazawa T, Chou KH (2009) Intercomparison of targeted observation guidance for tropical cyclones in the Northwestern Pacific. Mon Weather Rev 137-8:2471-2492

Yeh K-S, Côté J, Gravel S, Methot A, Patoine A, Roch M, Staniforth A (2002) The operational CMC-MRB global environmental multiscale (GEM) model. Part III: non-hydrostatic formulation. Mon Weather Rev 130:339-356

Zadra A, Roch M, Laroche S, Charron M (2003) The subgrid scale orographic blocking parameterization of the GEM model. Atmos Ocean 41:155-170

Zadra A, Buehner M, Laroche S, Mahfouf J-F (2004) Impact of the GEM model simplified physics on extratropical singular vectors. Q J R Meteorol Soc 130:2541-2569

Zadra A, Caya D, Côté J, Dugas B, Jones C, Laprise R, Winger K, Caron L-P (2008) The next Canadian regional climate model. La Physique au Canada 64-2:75-83 